\title{
Adaptivity based on error estimation for viscoplastic softening materials
}

\author{
Pedro Díez, Marino Arroyo and Antonio Huerta* \\ Departamento de Matemática Aplicada III, E.T.S. Ingenieros de Caminos, Universitat Politècnica de Catalunya, Spain
}

\begin{abstract}
SUMMARY
This paper focuses on the numerical simulation of strain softening mechanical problems. Two problems arise: (1) the constitutive model has to be regular and (2) the numerical technique must be able to capture the two scales of the problem (the macroscopic geometrical representation and the microscopic behaviour in the localization bands). The Perzyna viscoplastic model is used in order to obtain a regularized softening model allowing to simulate strain localization phenomena. This model is applied to quasistatic examples. The viscous regularization of quasistatic processes is also discussed: in quasistatics, the internal length associated with the obtained band width is no longer only a function of the material parameters but also depends on the boundary value problem (geometry and loads, specially loading velocity).

An adaptive computation is applied to softening viscoplastic materials showing strain localization. As the key ingredient of the adaptive strategy, a residual-type error estimator is generalized to deal with such highly non-linear material model.

In several numerical examples the adaptive process is able to detect complex collapse modes that are not captured by a first, even if fine, mesh. Consequently, adaptive strategies are found to be essential to detect the collapse mechanism and to assess the optimal location of the elements in the mesh. Copyright $(\mathbb{C} 2000$ John Wiley \& Sons, Ltd.
\end{abstract}

KEY WORDS: adaptivity; error estimation; pollution error; viscoplastic regularization; softening; localization

\section{INTRODUCTION}

Adaptive mesh refinement in strain softening problems has received important attention in the last decade. The presence of two well-differentiated length scales in such problems seems to indicate that adaptive remeshing strategies, in a general sense, are the natural approach. Recall that the spatial interpolation of the primitive variables must describe both the macroscopic scale associated to the solid geometry and the micro-scale related to the shear band. For instance, Huerta and Pijaudier-Cabot [1] show for two different models, that in the localization area element sizes must be one order of magnitude smaller than the internal length if errors under 5 percent are desired. Moreover, a priori knowledge of the location of the localization area is sometimes not possible.

Adaptivity in finite element computations requires three main ingredients. The first one is an algorithm for increasing/decreasing the richness of the interpolation in a particular area of the

\footnotetext{
* Correspondence to: Departamento de Matemática Aplicada III, E.T.S. Ingenieros de Caminos, Universitat Politècnica de Catalunya, E-080304 Barcelona, Spain.
}

CCC 1082-5010/2000/020087-26\$17.50

Copyright (C) 2000 John Wiley \& Sons, Ltd. 
P. DÍEZ, M. ARROYO AND A. HUERTA

computational domain. For instance, a good mesh generator if $h$-refinement $[2,3]$ is used. Second, an error estimator or error indicator must be employed to locate where there is a need for refinement/de-refinement. And, third, a remeshing criterion must be used to translate the output of the error analysis into the input of the mesh generator, for instance, the distribution of desired mesh sizes.

These three steps are fundamental in adaptivity. However, only the second one will be discussed here because it is, probably, the critical one in softening problems. In fact, Ortiz and Quigley IV [4] presented an excellent discussion on the difficulties that have deterred an extensive application of adaptive methods in the context of strain localization: (1) path-dependent constitutive equations, and (2) error estimation relying, in statics, on the ellipticity of the equations which is lost at the inception of localization. Here, these two difficulties are overcome because: (1) an error estimator mathematically sound for path-dependent constitutive equations is employed, and (2) a well-known regularized model which precludes the loss of ellipticity is used. The main goal here, is to show that adaptive mesh refinement based on estimating the actual error is now possible for regularized problems. Any model maintaining ellipticity after the inception of the localization could be used. Moreover, the numerical examples show that adaptivity based on the error estimation is essential for accurate computations and, in certain problems, for capturing a realistic physical behaviour.

The study of localization in solids has received much attention and a number of different approaches have been devised to overcome the difficulties encountered during its analysis. A possible approach is to consider the tinit problem and consequentiy, jumps in the displacement field across surfaces plasticity where disc instance $[5-7]$. Ano tinuity in the displaden length is introduced to
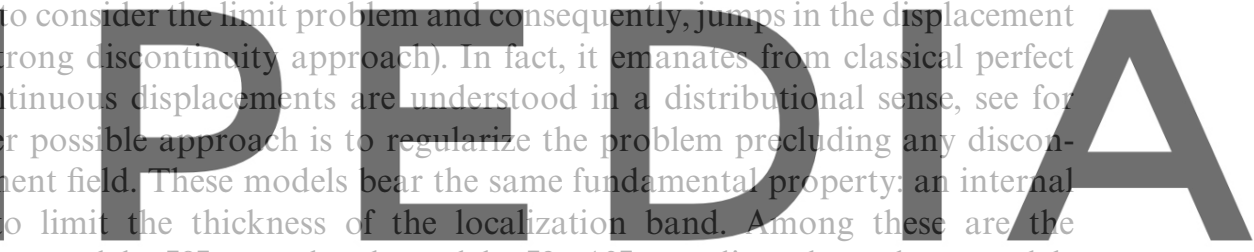

micropolar constitutive models [8], non-local models $[9,10]$, gradient-dependent models

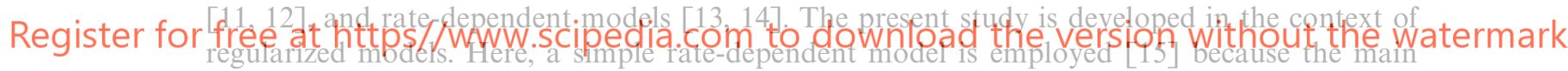
focus is on adaptivity.

The issue of a mathematically sound error estimator for path-dependent constitutive equations is crucial in this study. Adaptivity in softening problems has been associated to error indicators [16-18]. Error indicators are based on heuristic considerations while error estimators approximate a measure of the actual error in a given norm. In this work, a tool for assessing the error measured in the energy norm is proposed. The obtained approximation to the error is asymptotically exact, that is, tends to the actual error if the element size tends to zero $[19,20]$. In that sense, this tool is an error estimator.

Here, the assessment of the error associated with the finite element space discretization is the goal of the study. The influence of time discretization is out of the scope of this paper.

\section{PERZYNA VISCOPLASTICITY MODEL}

As noted previously, the Perzyna viscoplastic model is used for regularization [15, 21]. Thus, regularization is associated to viscous effects in the inelastic range. For clarity, this model is briefly reviewed in this section. 
In small-strain viscoplasticity, the strain rate $\dot{\boldsymbol{\varepsilon}}$ is decomposed into an elastic strain rate $\dot{\boldsymbol{\varepsilon}}^{\mathrm{e}}$ and a viscoplastic strain rate $\dot{\boldsymbol{\varepsilon}}^{\mathrm{vp}}$.

$$
\varepsilon=\varepsilon^{\mathrm{e}}+\varepsilon^{\mathrm{vp}}
$$

and the stress rate $\dot{\boldsymbol{\sigma}}$ is obtained as

$$
\dot{\boldsymbol{\sigma}}=\mathbf{C}:\left(\dot{\boldsymbol{\varepsilon}}-\dot{\boldsymbol{\varepsilon}}^{\mathrm{vp}}\right)
$$

where $\mathbf{C}$ is the elastic moduli tensor. Equations (1) and (2) are very similar to those of elastoplasticity, with plastic strain replaced by viscoplastic strain $\varepsilon^{\mathrm{vp}}$.

An expression for $\dot{\boldsymbol{\varepsilon}}^{\mathrm{vp}}$ is needed. For associative flow, the Perzyna model [15] takes

$$
\dot{\boldsymbol{\varepsilon}}^{\mathrm{vp}}=\gamma\left[\frac{\langle f\rangle}{\bar{\sigma}_{0}}\right]^{N} \frac{\partial f}{\partial \sigma}
$$

where $\gamma$ and $N$ are the material parameters of the model, $f$ is the Von Mises yield function which depends on the yield stress $\bar{\sigma}$ [22]. This yield stress is assumed to be a linear function of the equivalent viscoplastic strain $\kappa$, according to

$$
\bar{\sigma}=\left\langle\bar{\sigma}_{0}+h \kappa\right\rangle
$$

where $\bar{\sigma}_{0}$ is the initial yield stress and $h$ is the hardening (for $h>0$ ) or softening (for $h<0$, used here) parameter. The symbol $\langle f\rangle$ means that
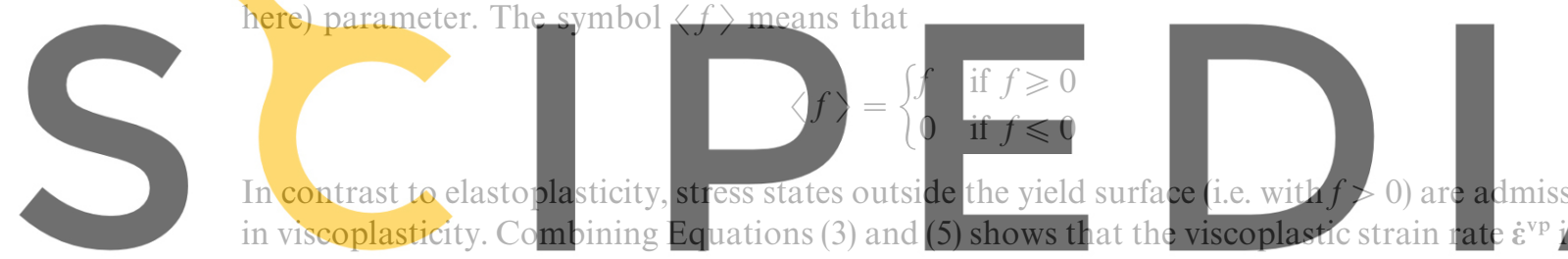

in viscoplasticity. Combining

general, non-zero. On the other

and, for stress states inside or on the yield surface, $\dot{\varepsilon}^{\mathrm{vp}}=0$ anc

[21]. The viscous effect is represented by the damper in the right, with viscosity $\bar{\sigma}_{0} / \gamma$, and softening is represented by the element on the left, which symbolizes the linear evolution of the yield stress (Equation (4)).

The Perzyna viscoplastic model has been used as a regularization technique in transient dynamic processes $[21,14]$. Here, it will be employed to regularize a quasistatic problem, see [23] for a discussion on dynamic and quasistatic analysis.

For dynamic analysis (i.e. inertia effects are considered) the width of the localization band can be predicted a priori as a function of material parameters [21]. It is independent of the loading velocity. The rationale is that the stress waves (those producing loading and unloading paths) travel at a celerity which depends only on material parameters. Thus, in dynamic analysis, the band width depends on the stress wave celerity and the viscous effects.

For quasistatic analysis processes (i.e. inertia terms are neglected) time does not appear explicitly in the momentum balance. However, time is still an independent variable of the problem because it is present in the constitutive relations (Equations (1)-(3)). Time has still its physical meaning and it is not, as in rate-independent elastoplasticity, a loading parameter (i.e. a pseudo-time). Note that for quasistatic problems the loading velocity has a crucial influence on the material response, see Figure 2 for a simple compression example. Thus, from a physical point 


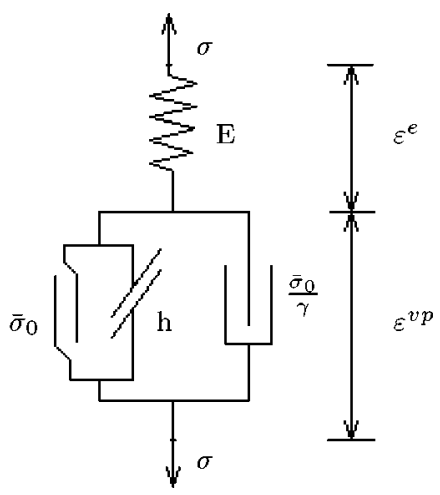

$$
\begin{aligned}
& \dot{\varepsilon}=\dot{\varepsilon}^{e}+\dot{\varepsilon}^{v p} \\
& \dot{\varepsilon}^{v p}=\frac{\gamma}{\bar{\sigma}_{0}}<\sigma-\bar{\sigma}> \\
& \dot{\sigma}=E\left(\dot{\varepsilon}-\dot{\varepsilon}^{v p}\right) \\
& \bar{\sigma}=\bar{\sigma}_{0}+\sqrt{\frac{5}{6}} h \varepsilon^{v p}
\end{aligned}
$$

Figure 1. One-dimensional rheologic scheme of Perzyna-type viscoplastic model $(N=1)$.

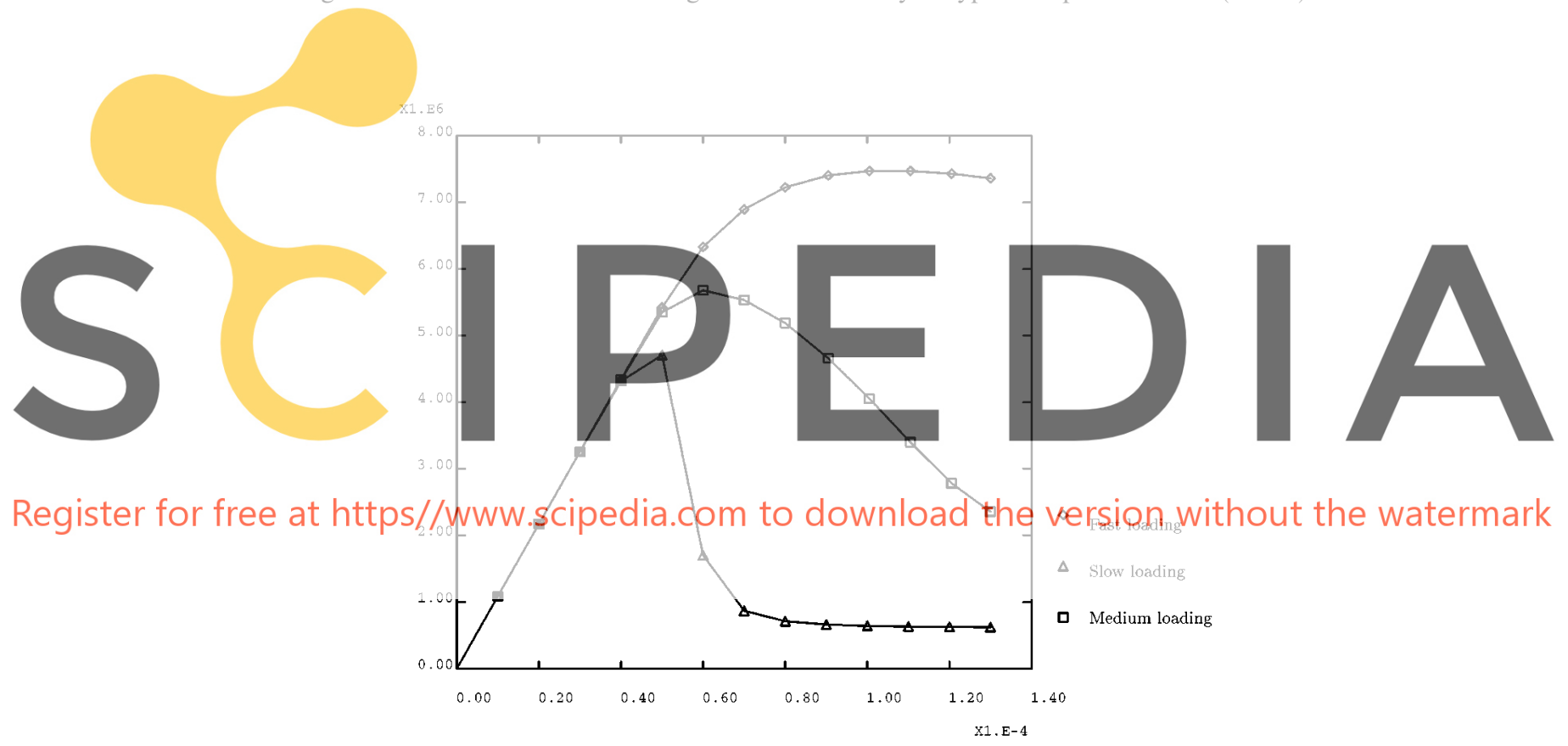

Figure 2. Influence of the loading velocity in the response of the Perzyna model in a quasistatic case (reaction versus imposed displacement).

of view, viscoplasticity in quasistatics is still a sound regularization technique (i.e. viscous effects and the associated regularization are still present). Section 4 illustrates this issue with several examples. In fact, viscoplasticity is an obviously sound regularization technique in a quasistatic analysis because a quasistatic problem is simply a dynamic problem where inertia terms are below the threshold of computational accuracy. Viscoplasticity is not employed here as a numerical strategy to avoid imposing the plastic consistency condition. It is assumed to be the 'real' material behaviour. It is not used as a numerical relaxation technique [24] designed to get the 
elastoplastic solution. In fact, for softening models, the steady-state limit (after zero loading velocity is reached) of a viscoplastic analysis does not coincide with an elastoplastic solution.

Nevertheless, in quasistatics, the velocity governing the loading and unloading is no longer the stress wave celerity but the external load velocity. Thus, the band width in quasistatics is not governed exclusively by material parameters, it depends also on the load velocity. In fact, in Reference [25] it is demonstrated that for one-dimensional problems the load velocity plays a crucial role in the resulting band width.

Another difference between dynamic and quasistatic viscoplastic analysis is related with the need and the influence of imperfections. It is well known that dynamic viscoplastic problems do not need an imperfection to induce localization, reflecting or incoming stress waves are usually employed to initiate localization. Quasistatic viscoplastic problems in simple domains, however, do need an imperfection to induce localization. In any case, both in dynamics and quasistatics, if an imperfection exists, the resulting band width may be affected by the imperfection in its vicinity, see $[21,26]$ for dynamic cases and $[27]$ or Section 4 for quasistatics.

\section{ERROR ESTIMATION AND ADAPTIVITY}

As discussed in the introduction, an error estimator is a key feature in any adaptive procedure. Many error estimators for linear standard problems have been introduced (see [28-30], for reviews). However, the generalization of these estimators to general nen-linear problems is

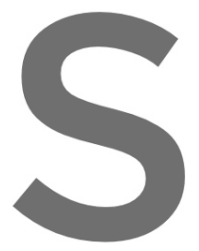
involved. This sectic which can be used in mathematical found performance is anal Using a mesh of cha
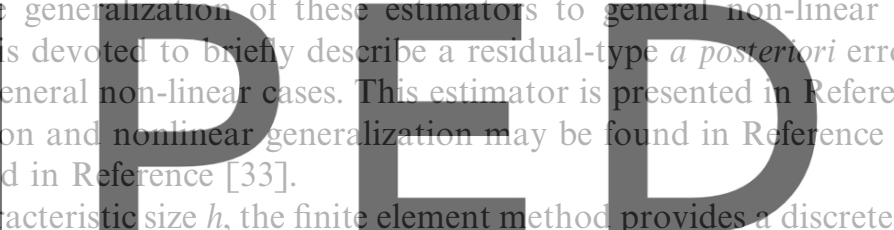
IA A equation where the unknown is the nodal displacement vector $\mathbf{u}_{h}$ :

\section{Register for free at https//www.scipedia.comint to. download the version without the (@atermark}

where $\mathbf{f}_{h}^{\text {int }}\left(\mathbf{u}_{h}\right)$ is the vector of nodal internal forces associated with $\mathbf{u}_{h}$ and $\mathbf{f}_{h}$ ext is the discretized external force term.

Once Equation (6) is solved, the solution $\mathbf{u}_{h}$ is affected by an error that has to be estimated. Since the actual displacements are unknown, the actual error cannot be computed. However, using a much finer mesh of characteristic size $\tilde{h}(\tilde{h} \ll h)$, the finite element method gives a new solution $\mathbf{u}_{\tilde{h}}$ which is much more accurate than $\mathbf{u}_{h}$ because the regularized model ensures that the Finite Element Analysis converges as the element size goes to zero. This solution can be taken as a reference solution and, consequently, the actual error can be fairly replaced by the reference error $\mathbf{e}_{\tilde{h}}$, the difference between $\mathbf{u}_{\tilde{h}}$ and $\mathbf{u}_{h}$.

Nevertheless, the determination of $\mathbf{u}_{\tilde{h}}$ (or $\mathbf{e}_{\tilde{h}}$ ) requires to solve an equation analogous to Equation (6) but in the finer mesh:

$$
\mathbf{f}_{h}^{\text {int }}\left(\mathbf{u}_{\tilde{h}}\right)=\mathbf{f}_{h}^{\text {int }}\left(\mathbf{u}_{h}+\mathbf{e}_{\tilde{h}}\right)=\mathbf{f}_{\tilde{h}}^{\text {ext }}
$$

This problem is much more expensive than the original one and it is unaffordable from a computational point of view.

In the remainder of this section a method for approximating $\mathbf{e}_{\tilde{h}}$ by low-cost local computations is presented. That is, instead of solving Equation (7), $\mathbf{e}_{\tilde{h}}$ is approximated by solving a set of local 


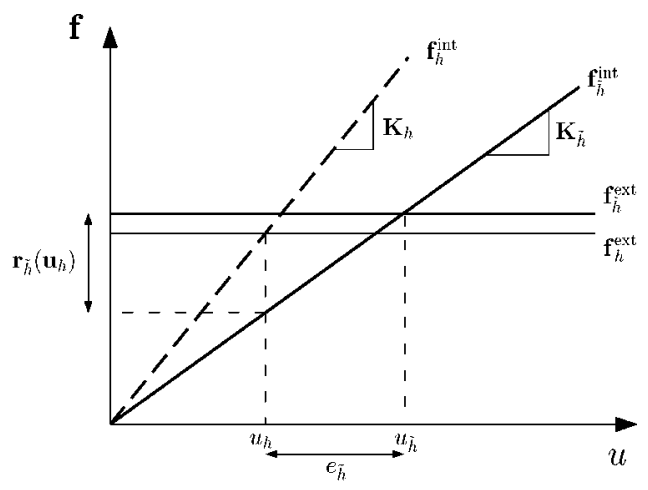

Figure 3. Graphic interpretation of reference error in linear problems.

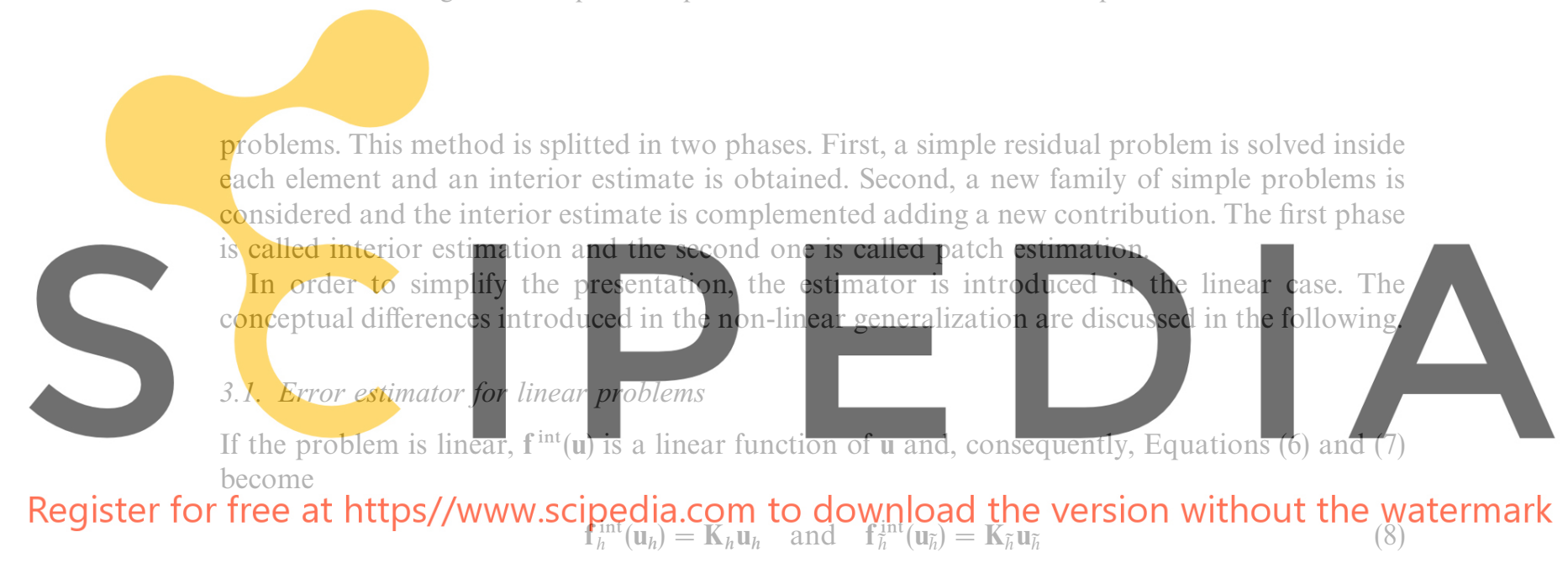

where $\mathbb{K}_{h}$ stands for the stiffness matrix associated with the coarse computational mesh and $\mathbf{K}_{\tilde{h}}$ stands for the stiffness matrix associated with the finer mesh. These equations can be easily manipulated and a linear equation for the reference error is found:

$$
\mathbf{K}_{\tilde{h}} \mathbf{e}_{\tilde{h}}=\mathbf{f}_{\tilde{h}}^{\text {ext }}-\mathbf{f}_{h}^{\text {int }}\left(\mathbf{u}_{h}\right)=:-\mathbf{r}_{\tilde{h}}\left(\mathbf{u}_{h}\right)
$$

where $\mathbf{f}_{h}^{\text {int }}\left(\mathbf{u}_{h}\right)$ is the internal force vector in the finer mesh associated with the solution $\mathbf{u}_{h}$ of the coarse mesh, and $\mathbf{r}_{h}\left(\mathbf{u}_{h}\right)$ is the residual.

Figure 3 shows a graphic illustration of the meaning of the reference error and its relation with the residual. The determination of the solutions, $\mathbf{u}_{h}$ and $\mathbf{u}_{\tilde{h}}$, can be seen as the determination of the intersection between the curve describing the evolution of the internal forces and the threshold of the discretized external forces (they are assumed to be independent of the solution). In the linear case the evolution of internal forces is described by straight lines and everything turns trivial. Figure 3 shows that reference error, $\mathbf{e}_{\tilde{h}}=\mathbf{u}_{\tilde{h}}-\mathbf{u}_{h}$, and the residual, $\mathbf{r}_{\bar{h}}\left(\mathbf{u}_{h}\right):=\mathbf{f}_{h}^{\text {int }}\left(\mathbf{u}_{h}\right)-\mathbf{f}_{\tilde{h}}^{\text {ext }}$, are related in terms of the stiffness matrix in the reference mesh, $\mathbf{K}_{\tilde{h}}$, as indicated in Equation (9). Although this illustration may appear trivial in the linear case, a variation of this figure provides a good understanding for the non-linear case. 


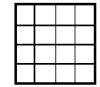

(a)

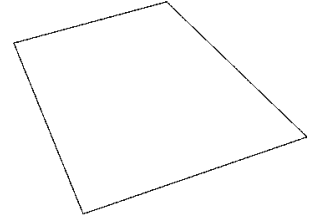

(b)

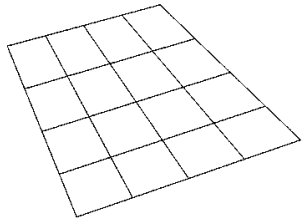

(c)

Figure 4. (a) Reference submesh mapped into (b) an element, to get (c) an elementary submesh.

3.1.1. Interior estimation. In order to avoid unaffordable computations (note that Equation (9) implies the resolution of a very large system of equations), the error estimation must be performed solving local problems. This is standard in residual-type error estimators. The natural partition of the domain in order to solve local problems is the set of elements of the 'coarse' computational mesh (denoted by $\Omega_{k}, k=1, \ldots$ ).

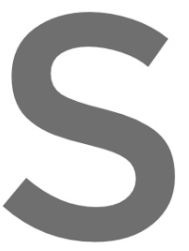

A finer reference 1 These elementary submo the elements of the

Then, the elementary of the original mesh. tions for the error. Most of residual-type error
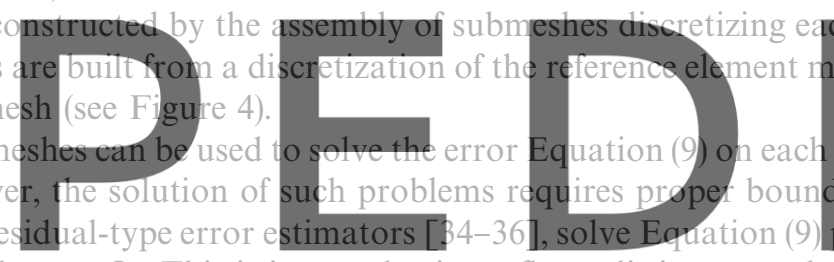

the error flux around each element $\Omega_{k}$. This is imposed using a flux splitting procedure which is

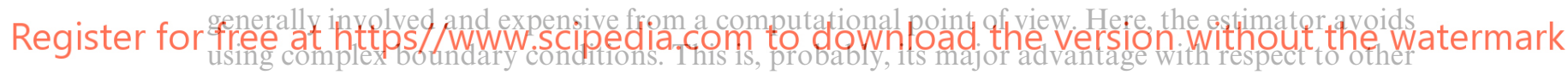
residual-type estimators. Trivial boundary conditions are imposed for both sets of local problems. Moreover, in contrast with other residual-type estimators the local problems are soived using an interpolation space based on $h$-refinement (easier to implement from a computational point of view) instead of the usual bubble spaces ( $p$-refinement).

In this work, the elementary problems are solved in a straightforward manner imposing the displacement error to vanish along the boundary of each element $\Omega_{k}$ (see $[31,32]$ ). That is Equation (9) is solved at element level and the error is prescribed to zero in all the boundary nodes of the elementary submesh. This choice is the simplest one for these local boundary conditions. This discrete local problem leads to a system of equations

$$
\mathbf{K}_{k}^{\mathrm{e}} \boldsymbol{\varepsilon}_{k}=-\mathbf{r}_{k}^{\mathrm{e}}
$$

where $\mathbf{K}_{k}^{\mathrm{e}}$ is the local stiffness matrix associated with the elementary submesh discretizing $\Omega_{k}$ and $\mathbf{r}_{k}^{\mathrm{e}}$ is the restriction of the residual to this element. The vector $\boldsymbol{\varepsilon}_{k}$ is an approximation to the restriction of the reference error $\mathbf{e}_{\tilde{h}}$ inside the element $\Omega_{k}$. In the linear case, the squared local energy norm of the interior estimate $\boldsymbol{\varepsilon}_{k}$ can be directly computed by

$$
\left\|\boldsymbol{\varepsilon}_{k}\right\|^{2}=\boldsymbol{\varepsilon}_{k}^{\mathrm{T}} \mathbf{K}_{k}^{\mathrm{e}} \boldsymbol{\varepsilon}_{k}=-\boldsymbol{\varepsilon}_{k}^{\mathrm{T}} \mathbf{r}_{k}^{\mathrm{e}}
$$




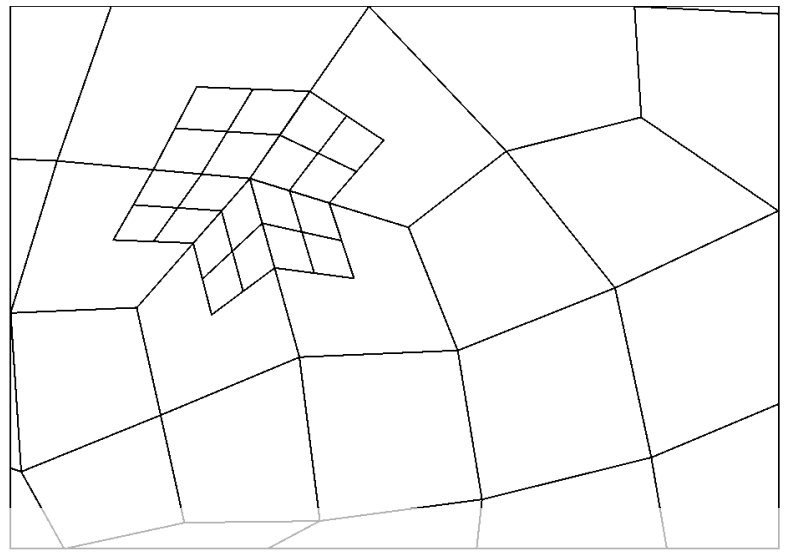

Figure 5. Patch submesh centred in a node of the computational mesh.

It is worth remarking that the squared norm of the error is simply computed as the scalar product

of two vectors: the estimated displacement error and the residual. This avoiding extra computa-

tions involving the

up a global estimate
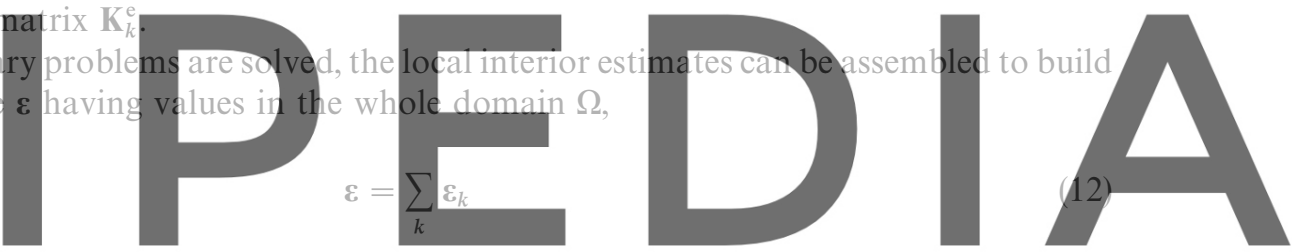

The global norm of \& can be easily computed using the local estimates

Register for free at https//www.scipedia.com to download the version without the watermark

$$
\|\varepsilon\|^{2}=\sum_{k}\left\|\varepsilon_{k}\right\|^{2}
$$

The previous choice of the homogeneous boundary condition implies that $\|\boldsymbol{\varepsilon}\| \ll\left\|\mathbf{e}_{\tilde{h}}\right\|$. The reference error $\mathbf{e}_{\tilde{h}}$ is, most probably, non-zero along the element edges, thus $\boldsymbol{\varepsilon}$ may be a poor approximation to $\mathbf{e}_{\tilde{h}}$. In other words, the information contained in the flux jumps is ignored.

3.1.2. Patch estimation and complete estimate. Once the interior estimate is computed, it is necessary to add the contribution of the flux jumps, that is, to improve the error estimation by adding non-zero values in the element boundaries. This can be done following the same idea of the interior estimation, precluding the direct computation of flux jumps and avoiding any flux splitting procedure.

The interior estimate is based on solving local problems in the elements. This has been done because using the finite element method, elements are the natural partition of the domain $\Omega$. In this second phase, a different set of subdomains, called patches, is considered, each one overlapping a few number of elements and covering a part of the elementary boundaries. Using the elementary submeshes of Figure 4, the most natural choice for patch subdomains is to associate 
them with the nodes of the mesh: each patch is associated with a node and includes a fourth of every element where the node belongs (see Figure 5 for an illustration).

The idea is to use this new partition to define new local problems for the error and to solve them. Local boundary conditions are imposed in the same fashion as in the previous phase (interior estimate); that is, homogeneous Dirichlet boundary conditions on the whole boundary of each patch. A new approximation to the error is obtained. This new approximation takes non-zero values in the boundary of the elements, where the interior estimate $\varepsilon$ vanishes. In order to solve these problems each patch must be discretized by a patch submesh. The discretization of Equation (9) using this patch submesh leads to a system of equations analogous to Equation (11). Since patches cover the edges of the elements, the restriction of the righthand-side residual term of Equation (9), accounts for the residual associated with the flux jumps.

Using the patch estimate, local and global estimates can be computed following equations analogous to (11) and (13). The patch estimate must be forced to verify an additional restriction in order to be properly added to the previously computed interior estimates: it must be orthogonal to the global interior estimate $\varepsilon$. This is done to cancel the component of the patch estimate that has already been included in the interior estimate, see $[32,33]$ for details. This orthogonality condition can be easily implemented modifying the system of equations that gives the patch estimate, using the Lagrange multiplier technique [37].

Thus, the interior estimate $\varepsilon$ is completed and a new approximation to $\mathfrak{e}_{\tilde{h}}$ is found. Since it is
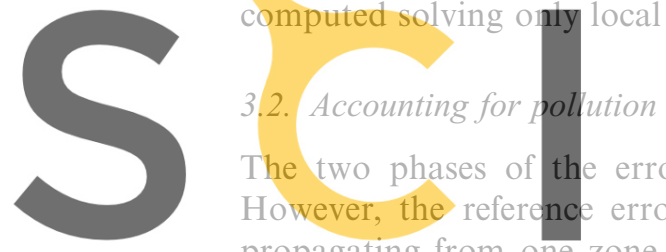

propagating from one zone
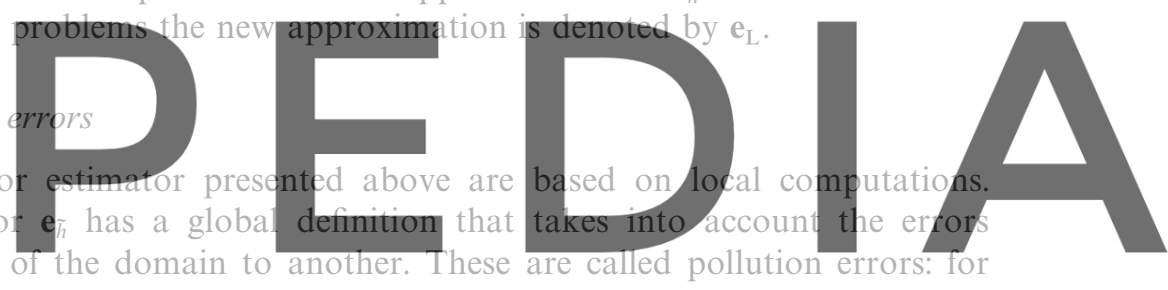

instance, corner singularities on the boundary pollute the solution in the whole domain introduc-

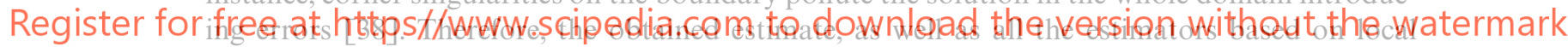

computations, ignore the existence of pollution errors.

Summing up the contribution of the interior and patch estimate an approximation to the error, $\mathbf{e}_{\mathrm{L}}$, based on local computations is found. But $\mathbf{e}_{\mathrm{L}} \neq \mathbf{e}_{\tilde{h}}$ and the difference, $\mathbf{e}_{\mathrm{G}}:=\mathbf{e}_{\tilde{h}}-\mathbf{e}_{\mathrm{L}}$ is a global error that cannot be approximated using local computations. The problem is now how to approximate $\mathbf{e}_{\mathrm{G}}$ by a low cost but global computation.

The vector $\mathbf{e}_{\mathrm{G}}$ is discretized in the reference mesh of element size $\tilde{h}$ (because both $\mathbf{e}_{\tilde{h}}$ and $\mathbf{e}_{\mathbf{L}}$ are defined over the fine mesh). Thus, its computation is expensive (equal to the cost of Equation (9). However, an approximation $\mathbf{e}_{\mathrm{G}}^{*}$ can be obtained over the coarse mesh. This approximation to the global error verifies the following system of equations (see [32]):

$$
\mathbf{K}_{h} \mathbf{e}_{\mathrm{G}}^{*}=-\mathbf{f}_{h}^{\text {int }}\left(\mathbf{e}_{\mathbf{L}}\right)
$$

The system of Equations (14) has the same matrix as the original Equation (6). Then, if a direct method has been used to solve Equation (6), the matrix is already factorized and the cost of solving Equation (14) is low. Note that, in order to compute the right-hand side term of Equation (14), the integrals over elements of the coarse computational mesh which define each component of $\mathbf{f}_{h}^{\text {int }}\left(\mathbf{e}_{\mathrm{L}}\right)$ require a function, $\mathbf{e}_{\mathrm{L}}$, defined on the finer reference mesh. 


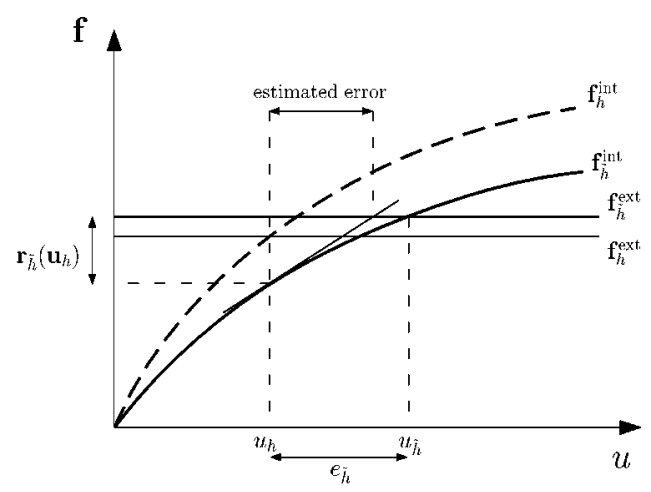

Figure 6. Graphic interpretation of reference error in non-linear problems and error estimation using tangent approximation.

3.3. Error estimator for non-linear problems

If the problem is non-linear, Equation (9) does not hold and the only available equation for the

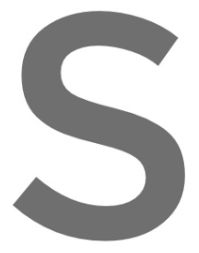
error is Equation (T

This is a general non-linear equation and must fact, this problem is equivalent to finding the $n$ However, here, the unknown $\mathbf{e}_{\tilde{h}}$ is small comp
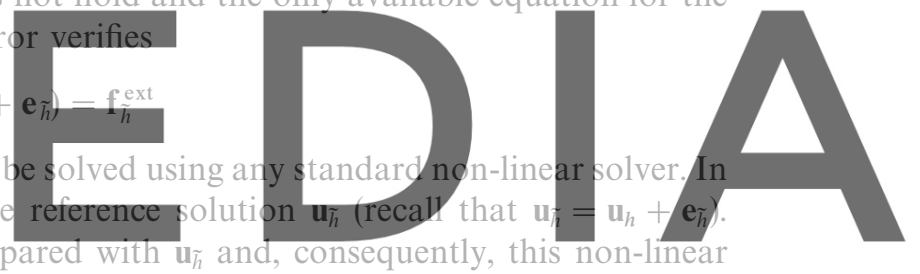
problem is much easier than the original one. Moreover, if this assumption is true and the tangent

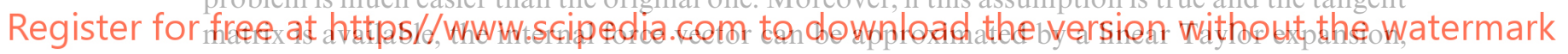
that is,

$$
\mathbf{f}_{\tilde{h}}^{\text {int }}\left(\mathbf{u}_{h}+\mathbf{e}_{\tilde{h}}\right) \approx \mathbf{f}_{\tilde{h}}^{\text {int }}\left(\mathbf{u}_{h}\right)+\mathbf{K}_{\mathrm{T}, \tilde{h}}\left(\mathbf{u}_{h}\right) \mathbf{e}_{\tilde{h}}
$$

where $\mathbf{K}_{\mathrm{T}, \tilde{h}}\left(\mathbf{u}_{h}\right)$ is the tangent matrix describing the linear behaviour of the system (discretized with the finer mesh) around $\mathbf{u}_{h}$.

Replacing Equation (15) in Equation (7), a new error equation is found:

$$
\mathbf{K}_{\mathrm{T}, \tilde{h}}\left(\mathbf{u}_{h}\right) \mathbf{e}_{\tilde{h}}=-\mathbf{r}_{\tilde{h}}\left(\mathbf{u}_{h}\right)
$$

where $\mathbf{r}_{h}\left(\mathbf{u}_{h}\right)$ is the residual, following the definition given in Equation (9). It is worth noting that Equation (16) is linear and has exactly the same structure of Equation (9).

Following the idea of the graphic illustration of Figure 3, Figure 6 shows how the non-linear case can be treated using a tangent approximation. The reference error is approximated using a tangent approximation of the curve representing the behaviour associated with the finer mesh. In this case, due to the non-linearity, the curves describing the behaviour of the internal forces are not straight lines.

The main idea of the generalization of the error estimator to non-linear cases is to reproduce the same structure of the linear case with a different equation for the error. Thus, again in this 
case, the previous idea is used and the estimation of the error is splitted in two steps. First, elementary problems are solved with null error boundary conditions, and an interior estimate is computed. Second, the restriction of Equation (16) to each one of the patches is solved. Moreover, the estimation of the global (pollution) error can also be reproduced in this context. In fact, once the error is set as the solution of the linear equation (16), the structure and the philosophy of the linear estimator can be fully respected and, consequently, the non-linear generalization inherits all the properties of the linear counterpart.

Recall that, even in this non-linear case, the energy of the solution may be measured using a simple scalar product: $\left\|\boldsymbol{\varepsilon}_{k}\right\|^{2}=-\boldsymbol{\varepsilon}_{k}^{\mathrm{T}} \mathbf{r}_{k}^{\mathrm{e}}$. If the tangent approximation is used and the tangent matrix is symmetric positive definite, this energetic quantity is the measure associated with the tangent matrix and, consequently, has a precise physical meaning.

\subsection{Adaptivity based on error estimation}

Once the error is estimated, the adaptive procedure requires a remeshing criterion in order to generate the input for the mesh generator. The error estimator furnishes local measures of the error in each element, that is, $\left\|\mathbf{e}_{\tilde{h}}\right\|_{k}$ for $k=1, \ldots$. This set of numbers describes the spatial distribution of the error. The input of a mesh generator is a distribution of desired element size in the computational domain. Generally, this is described by the desired element size in each element of the current mesh, that is, $\hat{h}_{k}$ for $k=1, \ldots$. Thus, a remeshing criterion is required to translate $\left\|\mathrm{e}_{\tilde{h}}\right\|_{k}$ into $\hat{h}_{k}$.

Different remeshing criteria have been defined [39-42] leading to quite different optimal meshes. This is because the underlying optimality criteria are different. In fact, all these remeshing criteria tend to equidistribute the error in some sense. The choice of the error function that has to be uniform is related with the underlying optimality criterion. In the examples presented in Section 4, two remeshing criteria are used and it is shown how the obtained meshes are very different.

In the following, the quadrilateral mesh generator developed by Sarrate [43] is used. This mesh generator supplies excellent unstructured meshes, both ensuring the prescribed element size and the regularity of the elements.

\section{NUMERICAL EXAMPLES}

Two examples are presented in this section. Both of them reproduce the compression of a plane strain rectangular specimen. In order to induce the strain localization in the specimen, some imperfection must be introduced. Typically, these imperfections are introduced by either a weaker element or a geometric imperfection $[14,21]$. Here, geometric imperfections are used: circular openings inside the material create a weaker zone in the specimen. The difference between the two presented examples is the number and the location of these circular openings. In the first example the specimen has one centred circular opening and, consequently, the two axes of symmetry allow to study only one fourth of the specimen (see Figure 7). In the second example, the specimen has two circular openings symmetric with respect to the centre. That allows to study only one-half of the specimen (see Figure 8). In both examples the tests are driven by imposing the velocity at the top of the specimen. 


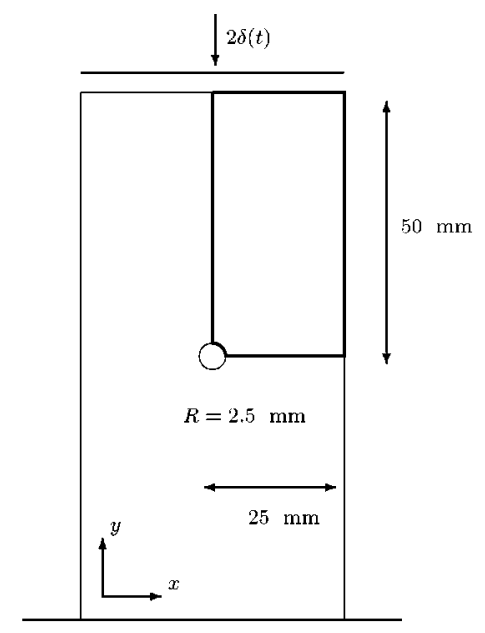

Two dimensional specimen with a circular opening

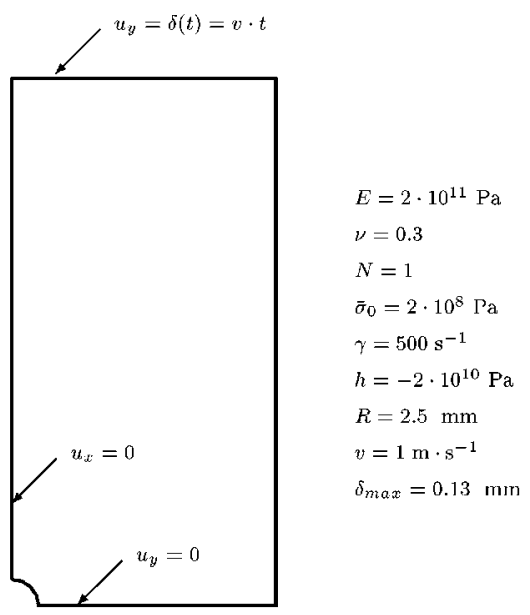

Computational domain

Figure 7. Rectangular specimen with one centred imperfection.
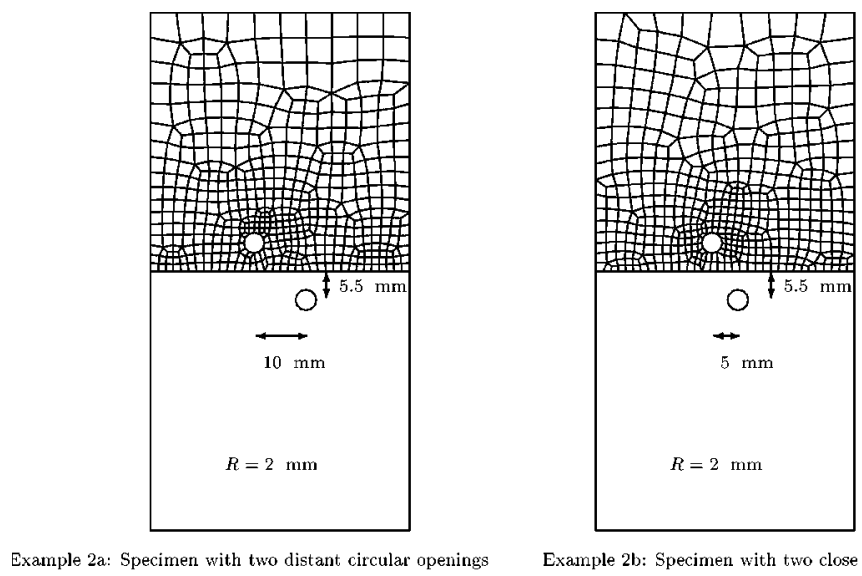

Example 2a: Specimen with two distant circular opening

Example 2b: Specimen with two close circular openings

Figure 8. Rectangular specimen with two symmetric imperfections.

\subsection{Rectangular specimen with one imperfection}

As shown in Figure 9, the collapse mechanism in this example is formed by two strain localization bands and softening behaviour is observed. In Figures 10 and 11 cross-sections of equivalent inelastic strain and Von Mises stress are shown. It can be seen that strain grows in the localization band along the loading process, but Von Mises stress remains almost constant once the failure mechanism is developed. Recall that all the deformation is localized along the band. Once the band is developed, the specimen behaves like two sliding rigid blocks: the material inside the band is practically flowing. 

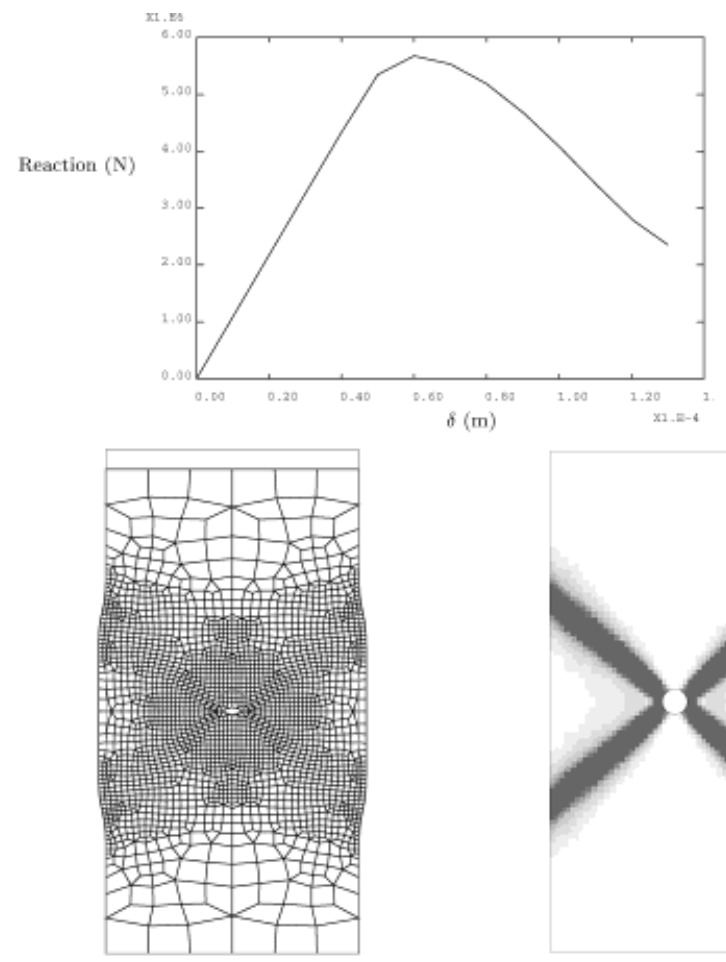

Mesh deformation amplified 15 times
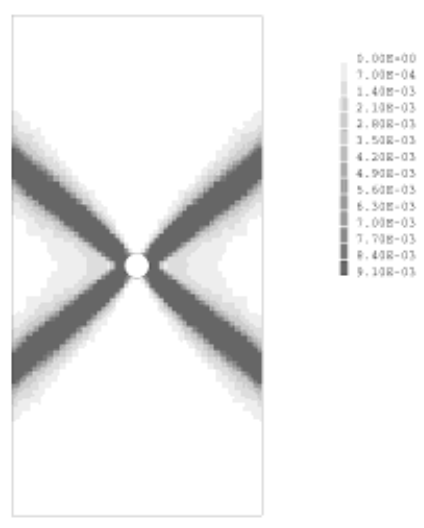

Figure 9. Description of Example 1.
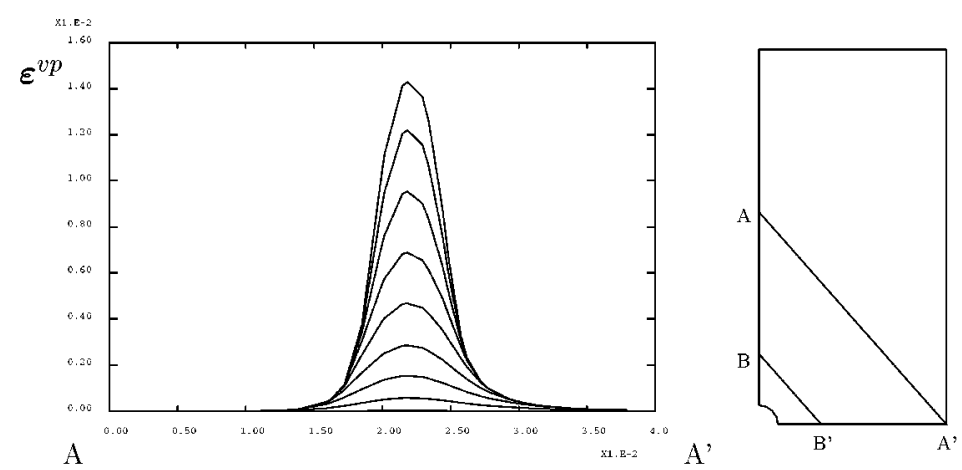

Figure 10. Evolution of the profile of the equivalent inelastic strain across the shear band along the normal direction $\left(\mathrm{A}-\mathrm{A}^{\prime}\right)$.

This simple example has been used to study the influence of the imperfection size and the loading velocity in the resulting localization band. First, for a given load velocity, three problems have been examined. The reference problem has an imperfection of radius $R=2.5 \mathrm{~mm}$ as shown in Figure 7. Two other problems have been studied with radii $R_{\text {small }}=1.25 \mathrm{~mm}$ and 


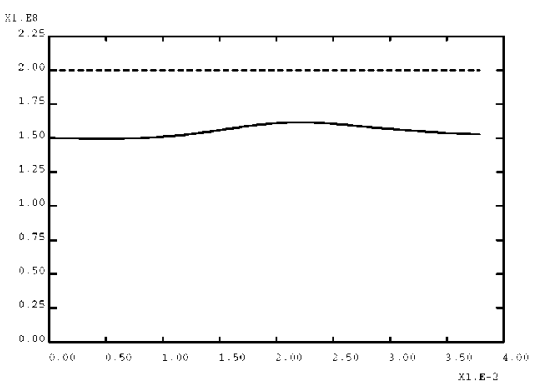

$\delta=0.04 \mathrm{~mm}$

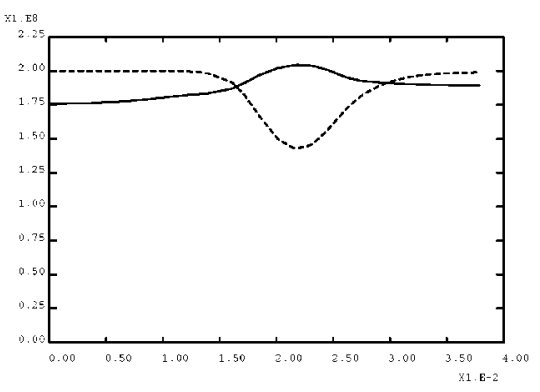

$\delta=0.08 \mathrm{~mm}$

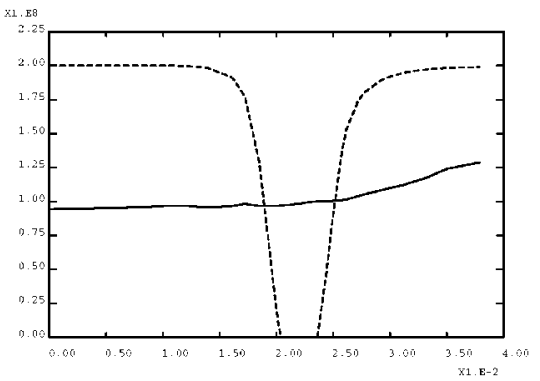

$\delta=0.12 \mathrm{~mm}$

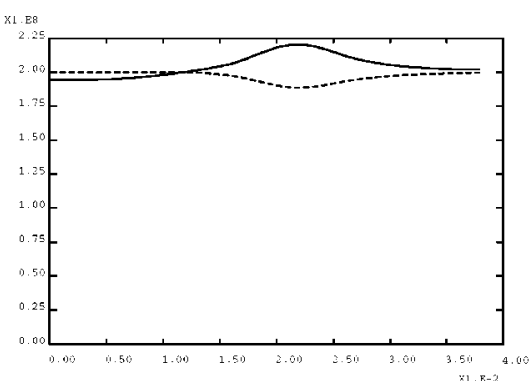

$\delta=0.06 \mathrm{~mm}$

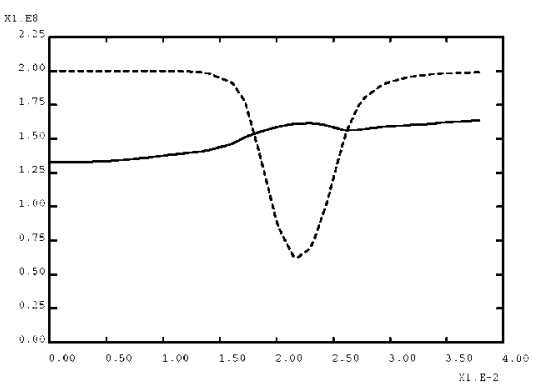

$\delta=0.10 \mathrm{~mm}$

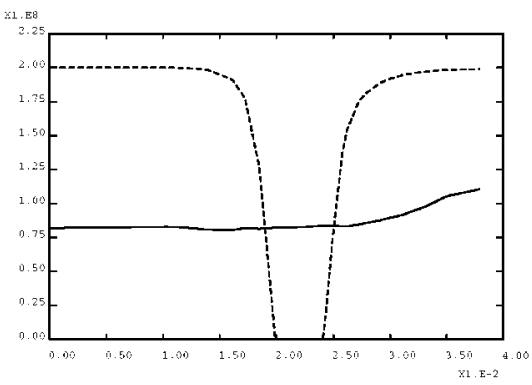

$\delta=0.13 \mathrm{~mm}$

Profiles along (A-A')

Figure 11. Evolution of the Von Mises equivalent stress (solid line) and the yield stress (dashed line) in the loading process.

$R_{\text {large }}=5 \mathrm{~mm}$. As shown in Figure 12, the specimen with the large imperfection has the same band width than the reference one. On the contrary, the specimen with the small imperfection has a narrower band in the neighbourhood of the imperfection. However, far enough from the imperfection, the width of the band is practically the same as in the other cases. In fact, if the loading process with the small imperfection continues, when the imposed displacement is large 


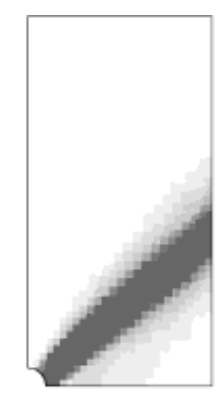

(a) $R \quad \delta=0.13 \mathrm{~mm}$

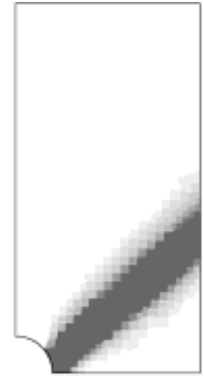

(b) $F_{\text {large }} \delta=0.13 \mathrm{~mm}$

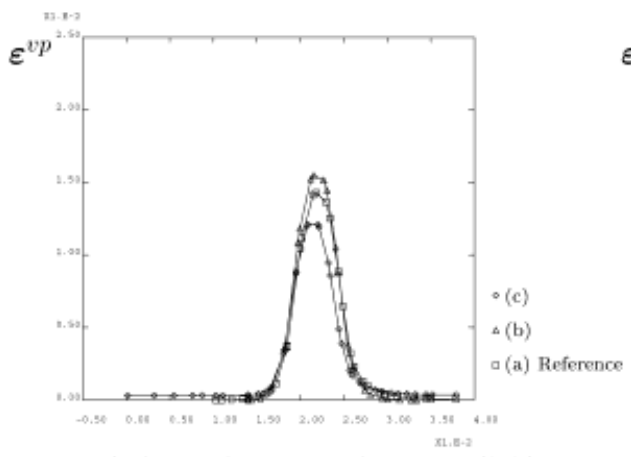

Profile far away from the imperfection along (A-A')

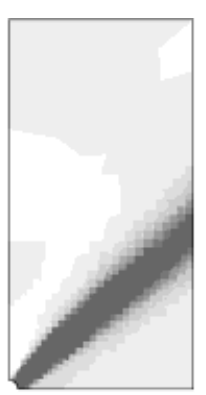

(c) $R_{\text {small }} \delta=0.13 \mathrm{~mm}$

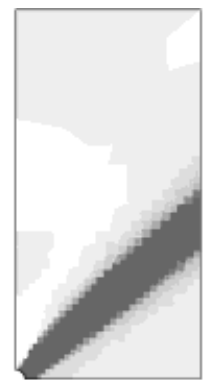

(d) $R_{\text {small }} \delta=0.19 \mathrm{~mm}$

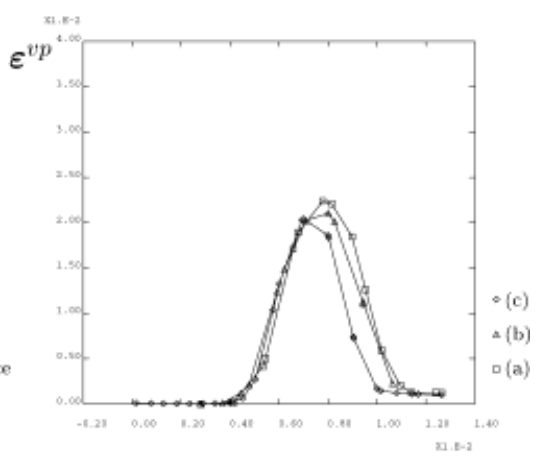

Profile near the imperfection along (B-B')

Figure 12. Influence of the imperfection size: contours and profiles of the inelastic strain.

enough (e.g. $\delta=0.19 \mathrm{~mm}$, see Figure 12) the band width is practically the same as the one obtained with larger imperfections. Thus, the imperfection size plays a role in the determination of the band width only if it is small. Moreover, this influence is only local and disappears at large load levels.

Second, the specimen with $R=2.5 \mathrm{~mm}$ has been studied with different loading velocities, see Figure 2. As expected (see discussion in Section 2 on viscoplastic regularization), when the load velocity decreases, the viscoplastic solution tends to the elastoplastic one. Thus, if loading velocity is very small the band width is very narrow and, from a practical point of view, the viscoplastic solution has the same problems as the elastoplastic: (1) pathological mesh dependence and (2) an almost vertical softening branch with the associated convergence difficulties, see [27] for further details.

An adaptive procedure has been used. First, the computations are carried out with a coarse almost uniform mesh. Then, the error is estimated at the end of the loading process. Using the estimated error distribution, a remeshing criterion and a mesh generator, a new mesh is created and the computations are carried out from scratch. This is repeated until the estimated error is below some acceptability requirements.

Firstly, two series of adapted meshes following the Li and Bettess [41] optimality criterion are presented. This remeshing criterion seeks uniform error distribution ( $\|e\|_{k}$ constant for every $k$ ). In the adaptive procedure of Figure 13 the error is estimated only locally (interior and patch 


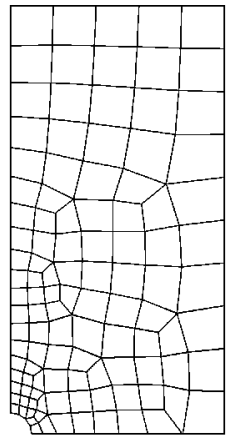

Mesh 0

110 elements

Global accuracy: $3.99 \%$

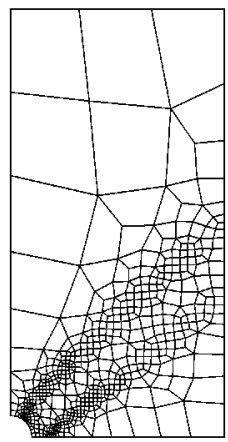

Mesh 3

561 elements

Global accuracy: $0.56 \%$

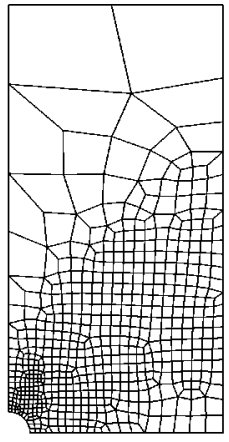

Mesh 1

590 elements

Global accuracy: $1.05 \%$

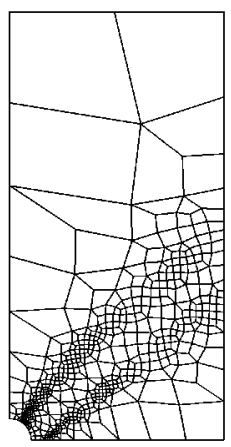

Mesh 4

564 elements

Global accuracy: $0.49 \%$

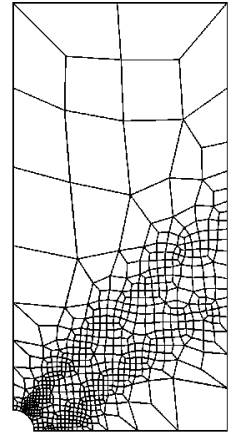

Mesh 2

611 elements

Global accuracy: $0.64 \%$

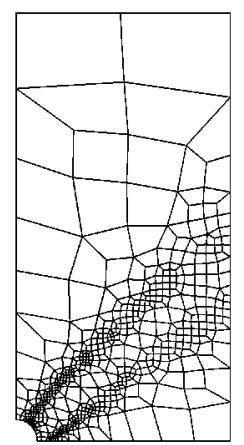

Final mesh with pollution errors 601 elements

Global accuracy: $0.49 \%$

Figure 13. Remeshing process using Li-Bettess criterion, without pollution errors, for a prescribed accuracy of 0.5 per cent.

estimate), in the series shown in Figure 14 pollution errors are also taken into account. The goal in both examples is to obtain an error below the 0.5 per cent. Discretizations corresponding to the local estimate have less elements than the ones obtained considering the local estimate have less elements than the ones obtained considering the pollution error. This is because the local error is lower and, consequently, a mesh with less elements suffices. However, the final distributions of elements are very similar. In fact, the pollution error is only relevant in the first mesh which is coarse and roughly uniform. Once the discretization is refined where it is needed (in particular in the vicinity of the singularities) the pollution effects are attenuated and become negligible. Considering pollution error does not make a big difference in the final results. This result was expected given the strong ellipticity of the problem. Figure 14 shows also the evolution of the error distribution along the adaptive procedure. The distribution of the error tends to be uniform, as expected.

In Figure 15 the adaptive procedure is carried out using a different remeshing criterion. Here, the uniform local accuracy (ULA) optimality criterion introduced in Reference [42] which is 


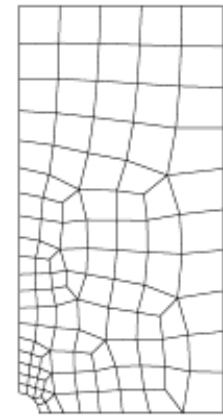

Mesh 0; 110 elements;

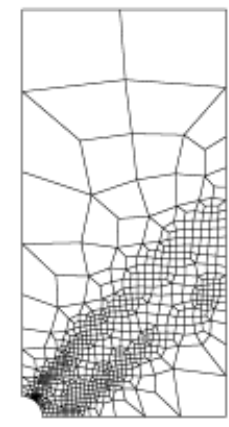

Mesh 2; 713 elements;

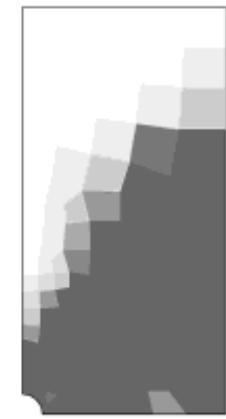

global accuracy; $4.07 \%$

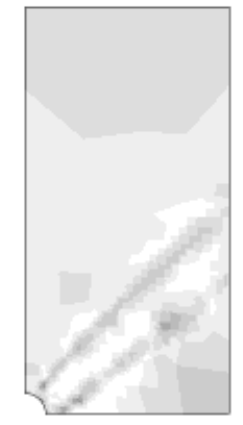

global accuracy: $0.56 \%$

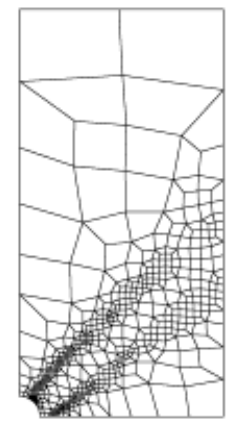

Mesh 4; 601 elements;

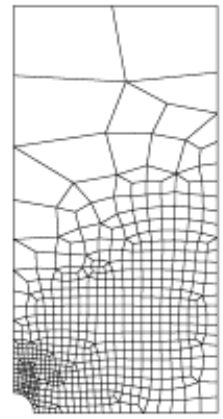

Mesh 1; 580 elements;

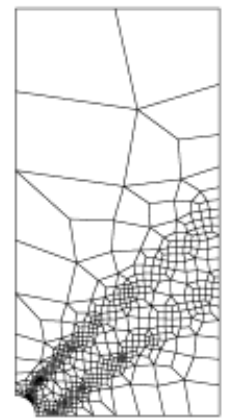

Mesh 3; 647 elements;

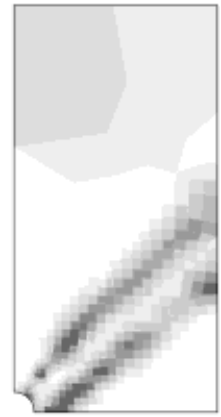

global accuracy: $1.08 \%$

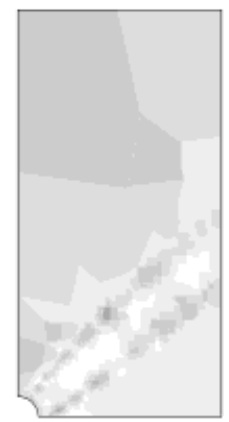

global accuracy: $0.48 \%$
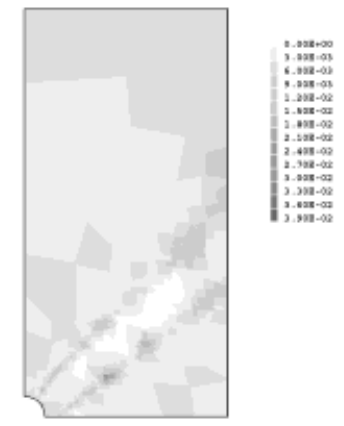

global accuracy: $0.49 \%$

Figure 14. Remeshing process using Li-Bettess and considering pollution errors for a prescribed accuracy of 0.5 per cent: succession of meshes and estimated error distributions.

based on obtaining a uniform distribution of local relative errors ( $\|e\|_{k} /\|u\|_{k}$ constant for every $k$ ) is used. In Figure 15 the accuracy maps (local relative error distribution) for the final mesh in Figure 14 is also shown. Using the ULA criterion the accuracy is almost uniform over the domain but it requires more elements than the Li and Bettess criterion. This result was expected because 


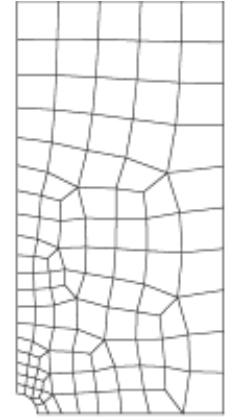

Mesh 0; 110 elements;

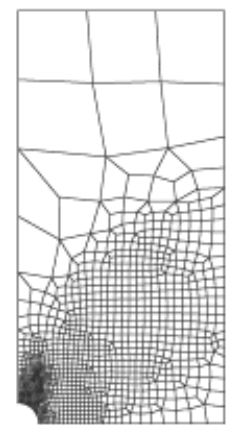

Mesh 1; 1206 elements;

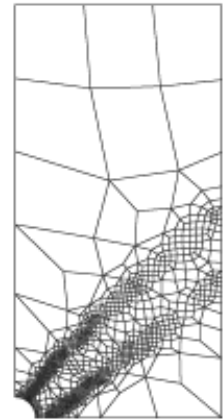

Mesh 2; 1977 elements;

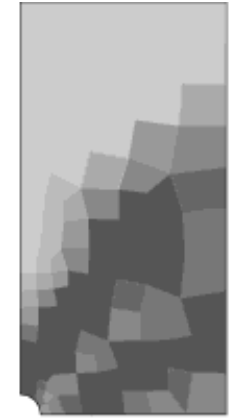

global accuracy: $4.07 \%$

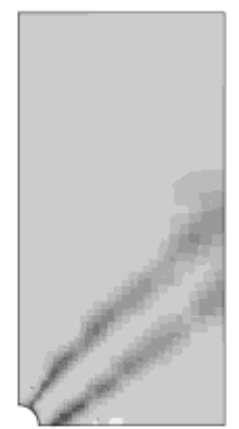

global accuracy: $0.64 \%$

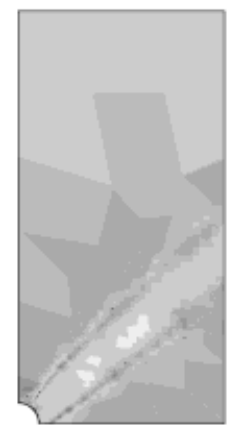

global accuracy: $0.25 \%$
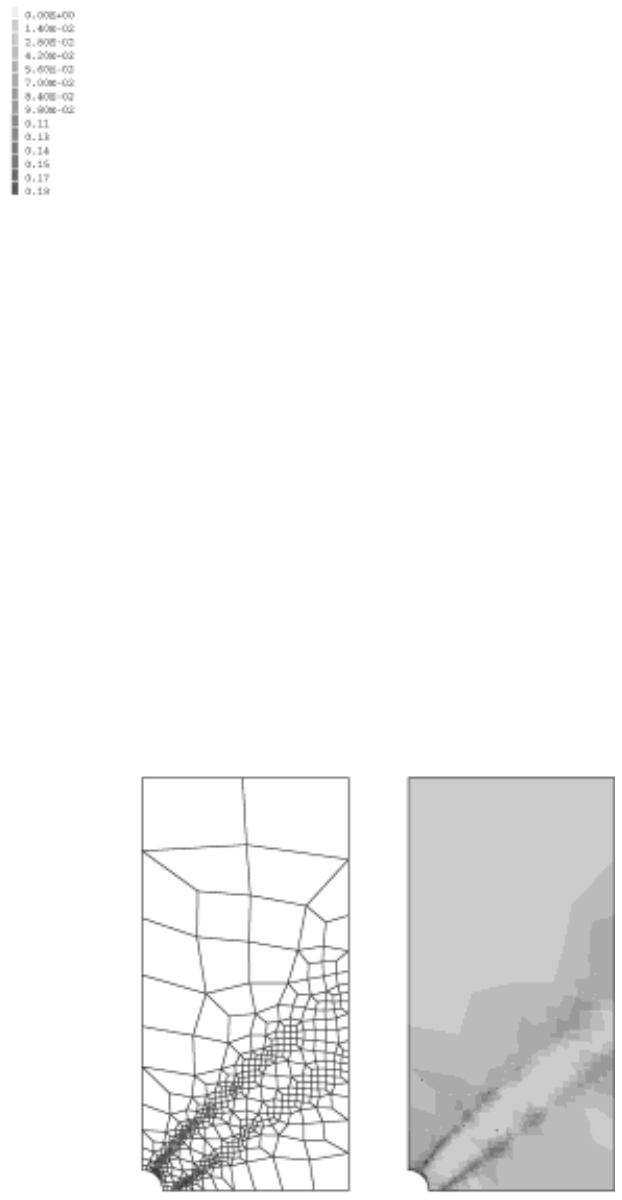

Li-Bettess final mesh and accuracy distribution 601 elements; global accuracy: $0.49 \%$

Figure 15. Remeshing process using ULA criterion and considering pollution errors for a prescribed accuracy of 0.5 per cent: succession of meshes, accuracy distributions and comparison with LB final mesh.

the $\mathrm{Li}$ and Bettess criterion is optimal in the sense that it produces the mesh with less elements given a globally acceptable error. The ULA criterion guarantees uniform accuracy, even locally, but it has a larger cost.

Note that the numerical examples confirm that the problem has been regularized because the behaviour of the solution does not vary along the remeshing process. The error estimation is robust in the sense that the adaptive procedure converges (the final mesh has the desired error). 


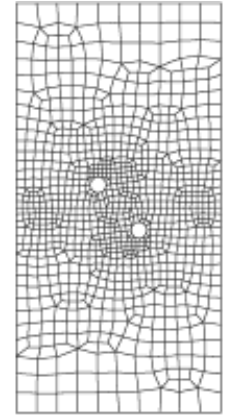

Mesh 0; 470 elements;

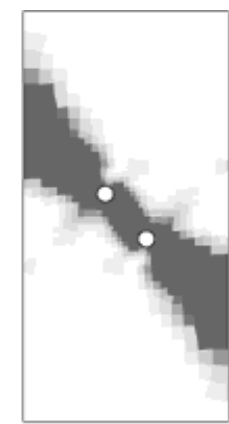

global accuracy: $6.75 \%$
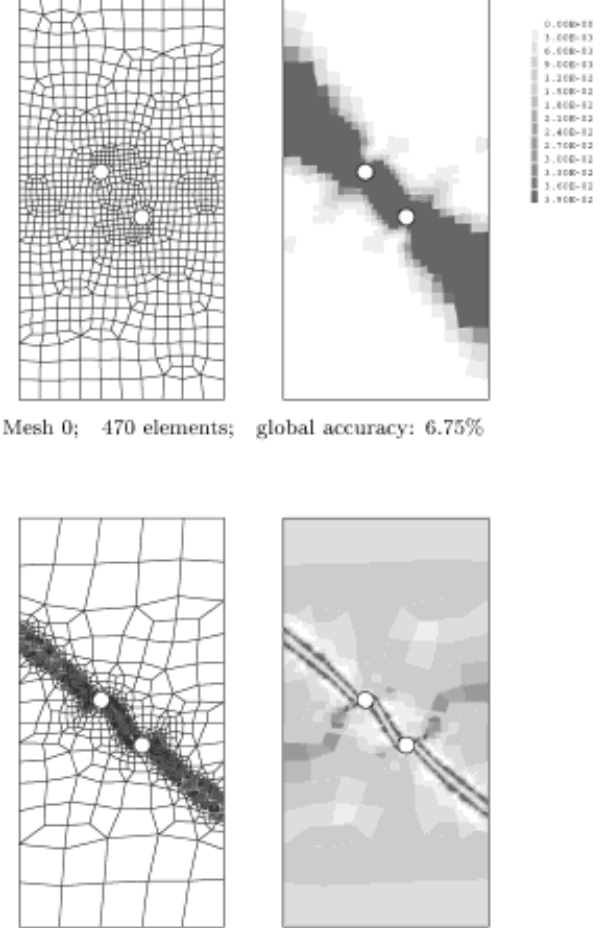

Mesh 2; 899 elements;

global accuracy: $2.28 \%$
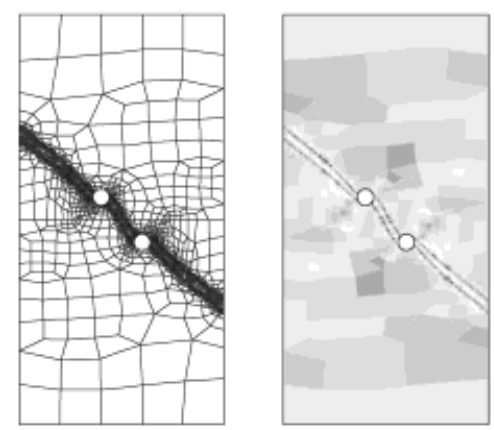

Mesh 4; 1660 elements; global accuracy: $1.62 \%$

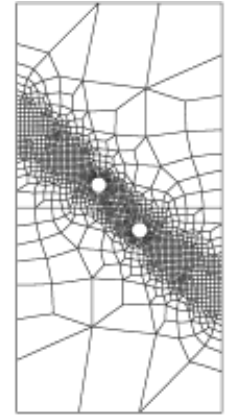

Mesh 1; 781 elements;

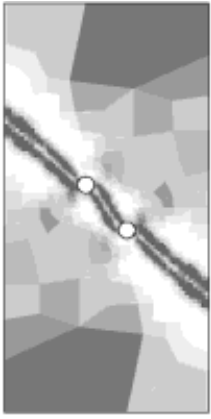

global accuracy: $3.00 \%$
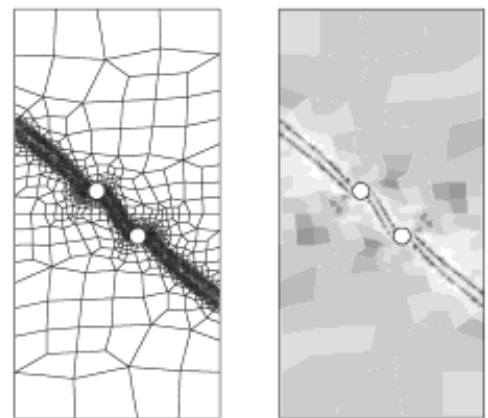

Mesh 3; 1274 elements; global accuracy: $1.97 \%$
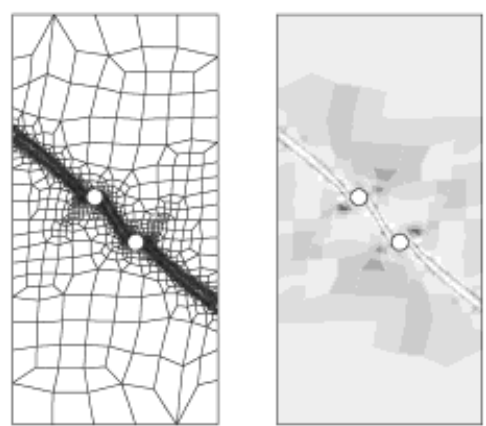

Mesh 5; 1888 elements; global accuracy: $1.35 \%$

Figure 16. Remeshing process using Li-Bettess for a prescribed accuracy of 1.5 per cent: succession of meshes and estimated error distributions.

\subsection{Rectangular specimen with two imperfections}

The mechanism of failure in this case is much more complex. In fact, it depends strongly on the position of the circular openings. Two cases are examined with different horizontal gaps between the openings, see Figure 8. In this example, the main concern is to capture the true collapse 

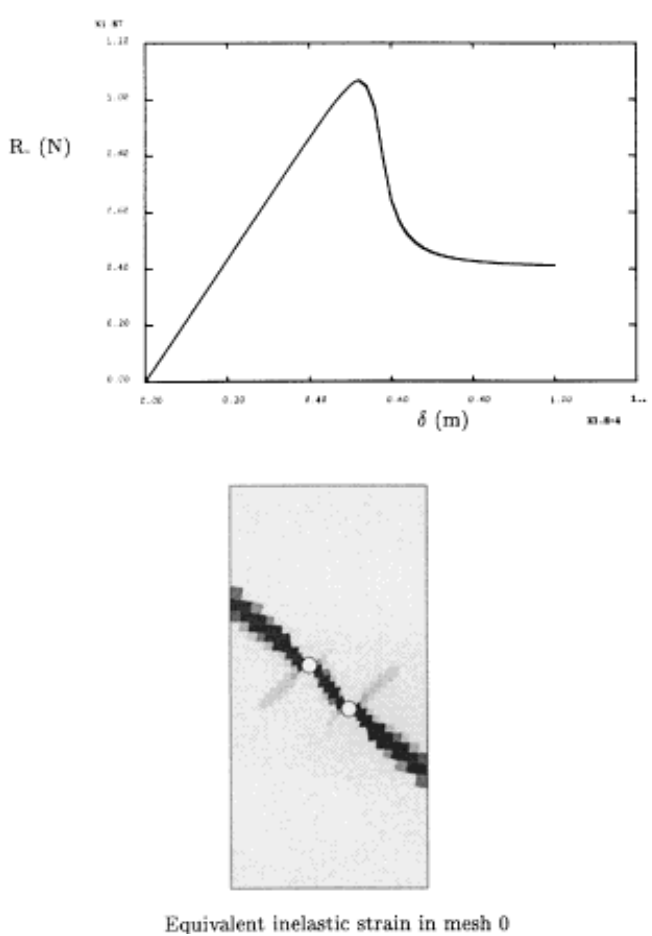

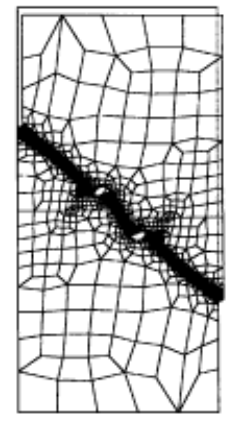

Mesh deformation amplified 10 times

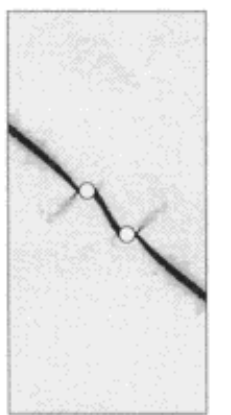

Equivalent inelastic strain in mesh 5

Figure 17. General solution for Example 2a: Reaction versus imposed displacement, deformation of mesh 5 and inelastic strain contours for meshes 0 and 5.

mechanism which minimizes the global energy. Thus, the goal is to reduce a global measure of the error. This is optimally done using the $\mathrm{Li}$ and Bettess remeshing criterion [41] and therefore is the one used for this example. Thus, the number of elements in the obtained meshes is minimum for a prescribed global accuracy even though the prescribed accuracy is not reached locally. If local accuracy is needed the ULA criterion, [42], should be used. In this case, the global mechanism will not vary but accurate stress distribution can be found in the localized area at the expense of larger computational cost.

\section{Example 2a. (Distant openings)}

If the horizontal distance between the circular openings is large enough, the behaviour is similar to the previous case. One shear band is developed aligned with the two openings. The remeshing process (see Figure 16) leads to a mesh with a large number of elements concentrated along a single shear band. Figure 17 shows the general behaviour of the solution: the softening force-displacement curve is similar to the previous case and the equivalent inelastic strain is concentrated along the shear band, both in the original and the final meshes of the remeshing process. That is, the captured collapse mechanism is the same in both meshes. 


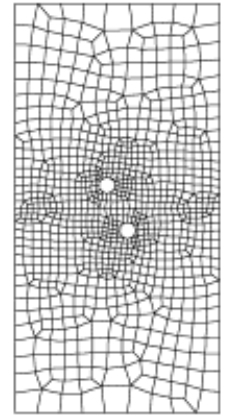

Mesh 0; 462 elements;

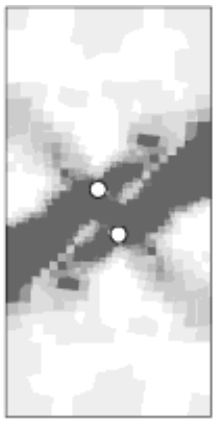

global accuracy: $4.99 \%$
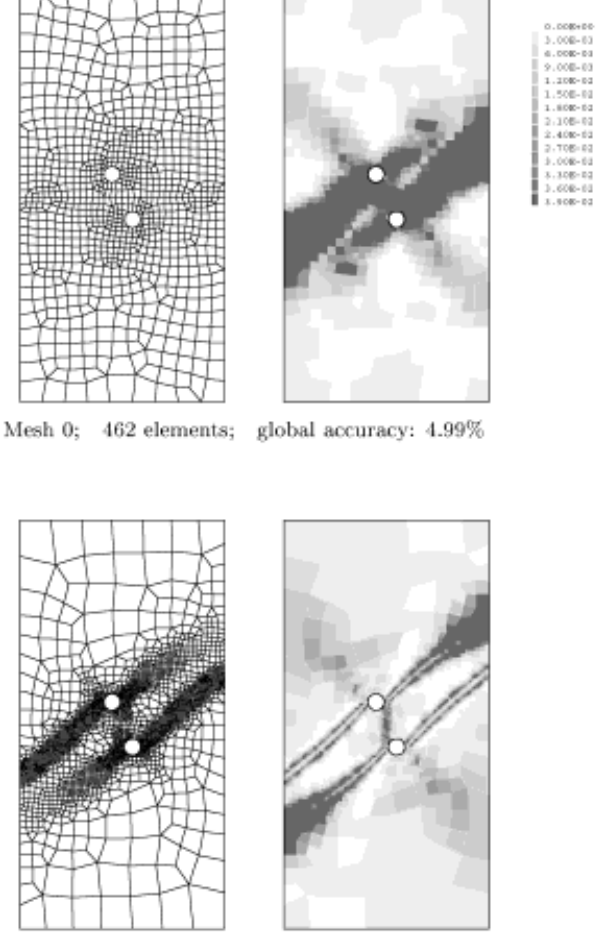

Mesh 2; 1641 elements;

global accuracy: $3.96 \%$
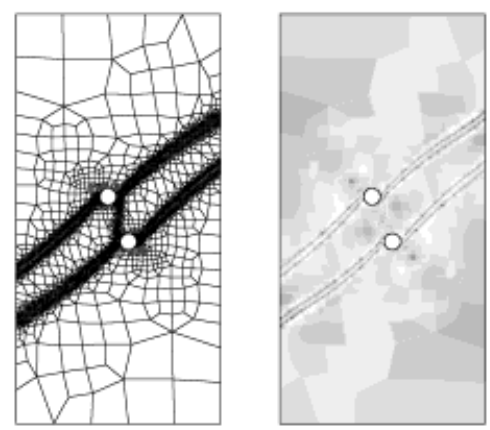

Mesh 4; 3200 elements; global accuracy: $1.73 \%$

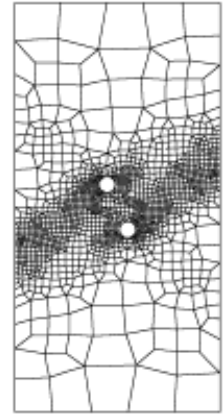

Mesh 1; 856 elements;

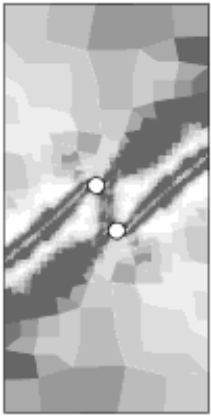

global accuracy: $4.00 \%$
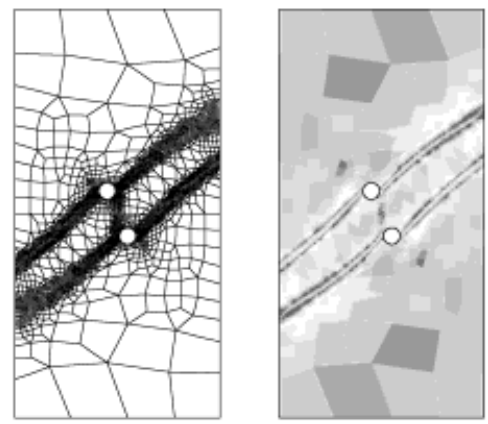

Mesh $3 ; 2235$ elements; global accuracy: $2.31 \%$
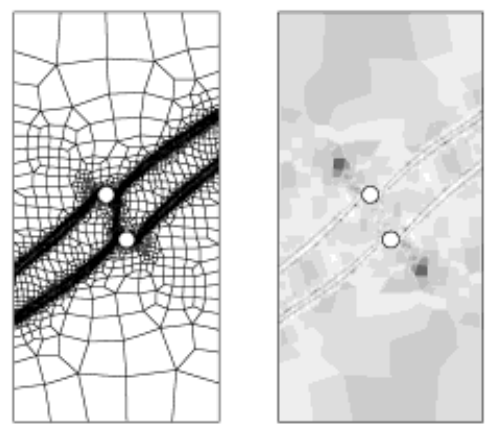

Mesh 5; 3307 elements; global accuracy: $1.49 \%$

Figure 18. Remeshing process using Li-Bettess for a prescribed accuracy of 1.5 per cent: succession of meshes and estimated error distributions.

\section{Example 2b. (Close openings)}

On the contrary, if the circular openings are closer, the behaviour of the solution is much more complex and the original mesh is not able to reproduce such a mechanism. 

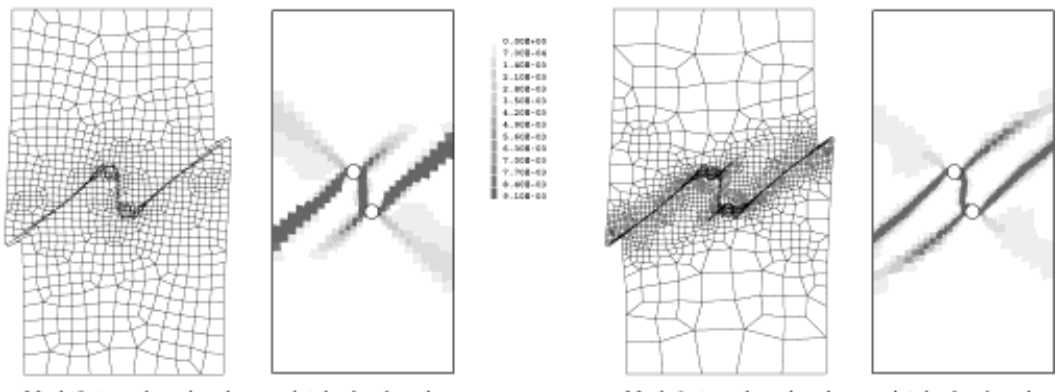

Mesh 0; two shear bands completely developed

Mesh 1; two shear bands completely developed
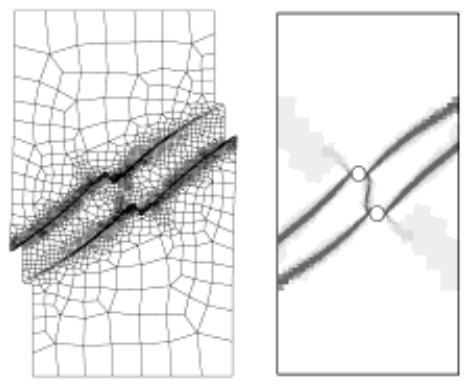

Mesh 2; four shear bands completely developed
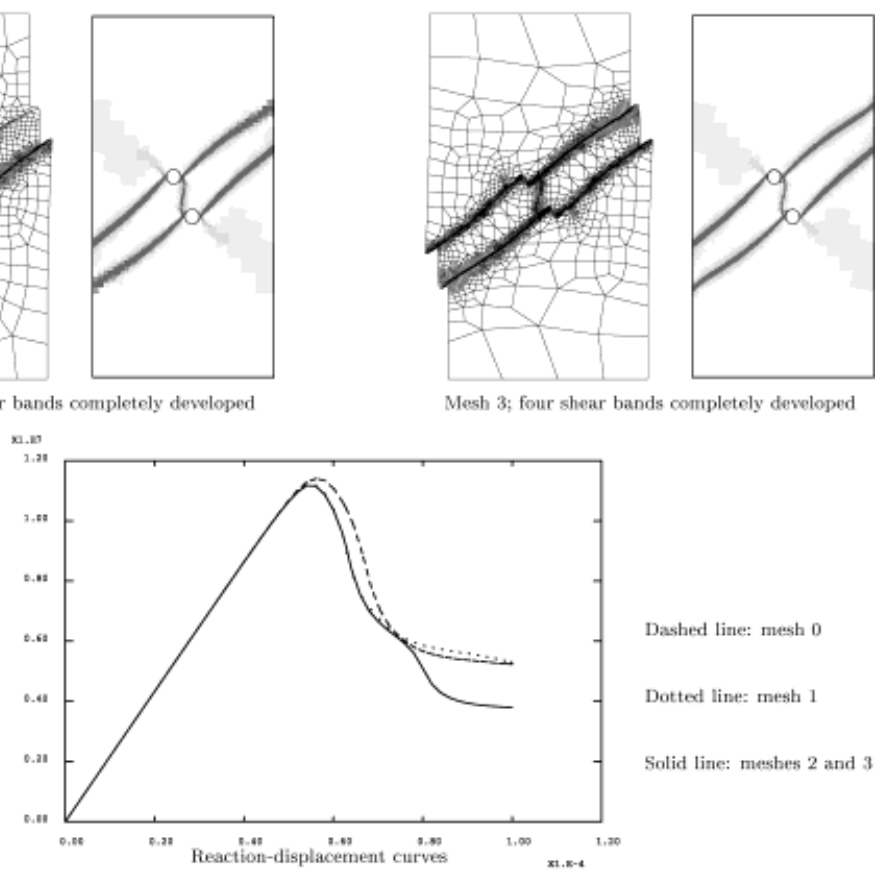

Mesh 3; four shear bands completely developed

Figure 19. Numerical bifurcation in the first meshes: mesh deformation amplified 40 times and equivalent inelastic strain contours.

Figure 18 shows the succession of meshes in this case. It is worth noting that, in the final mesh, according to the concentration of elements, two bands are developed. In fact, the resulting bands are not aligned with the imperfections (as in Example 2a), but have opposite inclination. Indeed Figure 19 shows how the computed equivalent inelastic strain and the deformation evolve along the remeshing process. Only after two remeshing steps the mesh captures two bands. In the previous meshes the discretization is not accurate enough and only one band is completely developed. Since large deformations are considered, once the first band evolves enough, the kinematic mechanism associated with this band locks: then a second band appears as a new 

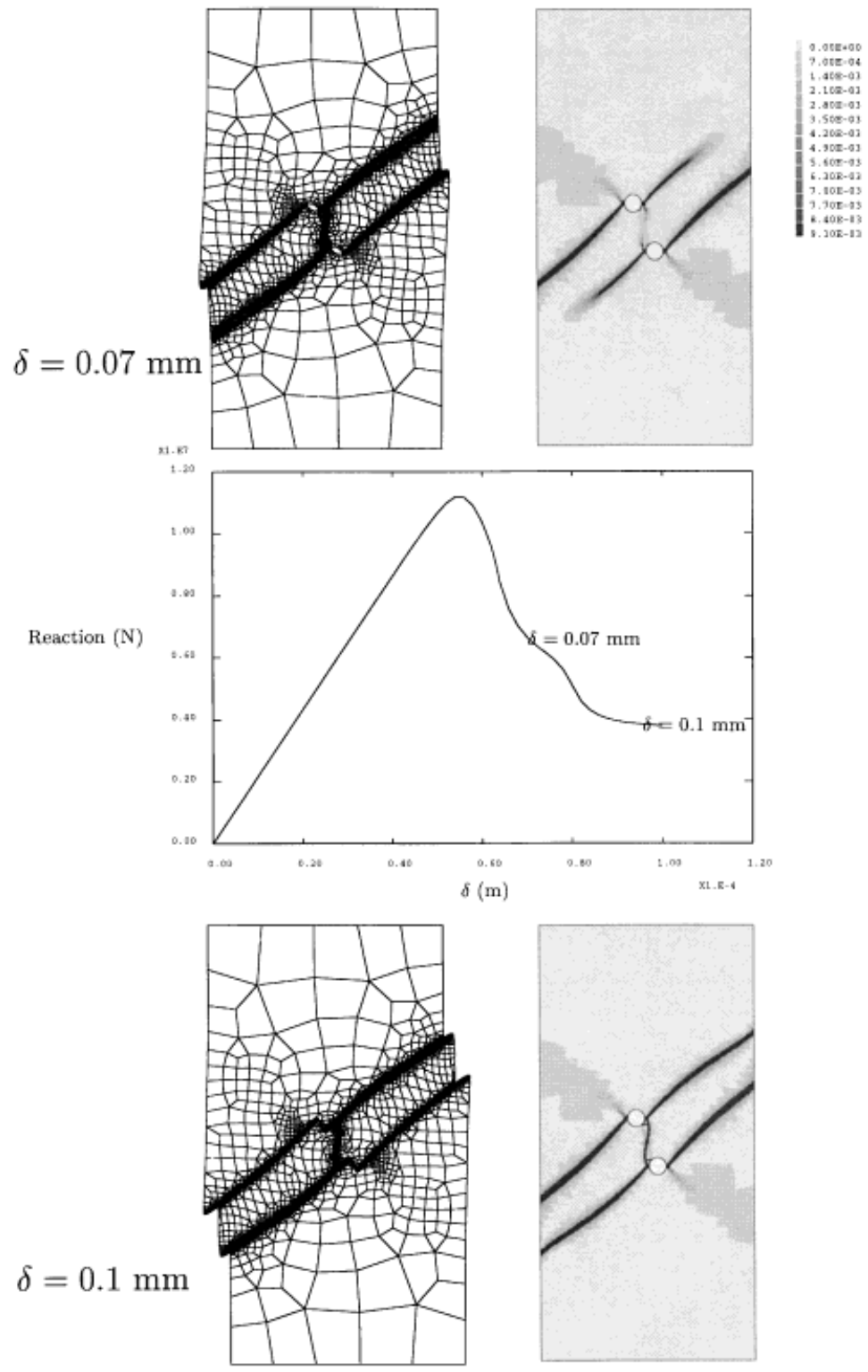

Figure 20. Two consecutive failure mechanisms for the final mesh: mesh deformation amplified 40 times and equivalent inelastic strain contours at two moments of the loading history.

deformation mode with less energy. Figure 19 shows also how the force-displacement curves for meshes 0 and 1 are qualitatively different from meshes 2 to 5 . In fact, the shape of the force-displacement curve for meshes 2-5 are practically identical and have two inflections in the descending branch. In Figure 20 it is shown how the inflections correspond to the formation of 
a new failure mechanism. The solution given by the last mesh is obviously more accurate than the original one because the energy of deformation (area under the force-displacement curve) is lower. In fact, since the error is controlled in energy norm, one can be sure that the actual curve, associated with the exact solution, is not too far from the obtained curve (the error in energy norm is less than 1.5 per cent and, consequently the difference of the area under the curves is less than 1.5 per cent). The first meshes are not able to reproduce the behavior of the actual solution because the elements along the later band (which develops in a further stage of the loading process) are too large and, consequently, the discretization is too stiff. Then, the size of the elements in this zone does not allow the inception of softening. On the contrary, once the remeshing process introduces small enough elements along the second band, the second mechanism can be captured.

Thus, this example demonstrates that adaptivity based on error estimation is an essential tool for the determination of a priori unpredictable final solutions. Without this adaptive strategy, the initial mesh (mesh 0 in Figure 18) and the resulting solution could be regarded as correct, and the second mechanism would not be detected.

\section{CONCLUDING REMARKS}

The Perzyna viscoplastic model has been used in order to obtain a regularized softening model allowing to simulate strain localization phenomena. This model has been applied to a quasistatic examples, where inertia terms are negligible. The viscous regularization of quasistatic processes has been discussed: the rate effects are still present and regularize the problem. However a difference between the dynamic and the quasistatic cases must be mentioned: in quasistatics, the internal length associated with the obtained band width is no longer only a function of the material parameters but also depends on the boundary value problem (geometry and loads, specially loading velocity).

An adaptive computation has been successfully applied to softening viscoplastic materials showing strain localization. As the key ingredient of the adaptive strategy, a residual-type error estimator has been generalized to deal with such highly non-linear material model. Moreover, this estimator has been designed in order to account for pollution errors. However, as expected, the pollution errors have been found to be negligible, specially in the refined meshes, with elements concentrated in the vicinity of the singularities.

In the numerical examples, the adaptive process is shown to be able to detect complex collapse modes that are not captured by a first, even if fine, mesh. This is specially interesting for softening localization problems, where small variations in the geometry of the problem may induce very different mechanical behaviour. In this situation the location of the localization band cannot be predicted a priori. For instance, in one of the examples the first mesh is not able to reproduce the two consecutive mechanisms captured with the final mesh. The second mechanism is associated with a second shear band appearing in a further stage of the loading process. However, if adaptivity is not used, the first mesh would be regarded as correct and one never would detect the second mechanism. In fact, even if some heuristic remeshing is done, based on solution obtained with the first mesh, the mesh would not be refined along the second shear band, that is, where it is needed to capture the second mechanism. On the contrary, if a remeshing strategy based on the error distribution is used, the elements are concentrated along this second band and the new mesh is able to reproduce the two mechanisms. Consequently, adaptive strategies based on error 
estimation are essential to detect the collapse mechanism and to assess the location of the elements in an optimal mesh.

\section{REFERENCES}

1. Huerta A, Pijaudier-Cabot G. Discretization influence on the regularization by two localization limiters. Journal of Engineering Mechanics ASCE 1994; 120(6):1198-1218.

2. Oden JT, Demkowicz L, Rachowicz W, Westermann TA. Toward a universal h-p adaptive finite element strategy, part 2. A posteriori error estimation. Computer Methods in Applied Mechanics and Engineering 1989, 77:113-180.

3. Zhu JZ, Hinton E, Zienkiewicz OC. Mesh enrichment against mesh regeneration using quadrilateral elements. Communications in Numerical Methods in Engineering 1993; 9:547-554.

4. Ortiz M, Quigley IV JJ. Adaptive mesh refinement in strain localization problems. Computer Methods in Applied Mechanics and Engineering 1991; 90:781-804.

5. Simo JC, Oliver J, Armero F. An analysis of strong discontinuities induced by strain-softening in rate-independent inelastic solids. Computational Mechanics 1993; 12:277-296.

6. Armero F, Garkipati K. An analysis of strong discontinuities in the multiplicative finite strain plasticity and their relation with the numerical simulation of strain localization in solids. International Journal of Solids Structure 1996; 33(20-22):2863-2885.

7. Larsson R, Runesson K. Element embedded localization band based on regularized displacement discontinuity. Journal of Engineering Mechanics, ASCE 1996; 122:402-411.

8. de Borst R. Simulation of strain localization: a reappraisal of the cosserat continuum. Engineering Computations 1991; 8:317-332.

9. Belytschko T, Bažant P, Hyun Y, Chang TP. Strain softening materials and finite element solutions. Computers and Structures 1986; 23:163-180.

10. Pijaudier-Cabot G, Bažant ZP. Nonlocal damage theory. Journal of Engineering Mechanics, ASCE 1987; 113:1512-1533.

11. de Borst R, Mühlhaus HB. Gradient dependent plasticity: formulation and algorithmic aspects. International Journal of Numerical Methods in Engineering 1992; 35:521-540.

12. Lasry D, Belytschko T. Localization limiters in transient problems. International Journal of Solids and Structures 1988; 24:581-597.

13. Sluys LJ, de Borst R. Wave propagation and localization in rate-dependent cracked medium. Model formulation and one dimensional examples. International Journal of Solids and Structures 1992; 29:2945-2958.

14. Belytschko T, Moran B, Kulkarni M. On the crucial role of imperfections in quasi-static viscoplastic solutions. Journal of Applied Mechanics, ASME 1991; 58:658-665.

15. Perzyna P. Fundamental problems in viscoplasticity. In Recent Advances in Applied Mechanics, Vol. 9. Academic Press: New York, 1966; 243-377.

16. Pastor M, Peraire J, Zienkiewicz OC. Adaptive remeshing for shear band localization problems. Archive of Applied Mechanics 1991; 61:30-39.

17. Yu J, Peric D, Owen DRJ. Adaptive finite element analysis of a strain localization problem for the elastoplastic cosserat continuum. Proceeding of the 3rd International Conference on Computational Plasticity, COMPLAS III, Owen DRJ et al. (eds). Pineridge Press: Swansea, 1992; 551-566.

18. Pijaudier-Cabot G, Bodé L, Huerta A. Arbitrary Lagrangian-Eulerian finite element analysis of strain localization in transient problems. International Journal for Numerical Methods in Engineering 1995; 38:4171-4191.

19. Babuška I, Durán R, Rodríguez R. Analysis of the efficiency of an a posteriori error estimator for linear triangular finite elements. SIAM (Society for Industrial and Applied Mathematics) Journal of Numerical Analysis 1992; 29:947-964.

20. Babuška I, Rodríguez R. The problem of the selection of an a posteriori error indicator based on smoothening techniques. International Journal for Numerical Methods in Engineering 1993; 36:539-567.

21. Sluys LJ. Wave propagation localization and dispersion in softening solids. Doctoral Thesis, Delft University of Technology, 1992.

22. Khan, Huang. Continuum Theory of Plasticity. Wiley: New York, 1995.

23. Rodríguez-Ferran A, Casadei F, Huerta A. ALE stress update for transient and quasistatic processes. International Journal for Numerical Methods in Engineering 1998; 43:241-262.

24. Owen, Hinton. Finite Elements in Plasticity. Pineridge Press: Swansea, 1980.

25. Needleman A. Material rate dependence and mesh sensitivity in localization problems. Computer Methods in Applied Mechanics and Engineering 1988; 67:69-85.

26. Wang WM, Sluys LJ, De Borst R. Interaction between material length scale and imperfection size for localization phenomena in viscoplastic media. European Journal of Mechanics A/Solids 1996; 15(3):447-464. 
27. Arroyo M, Díez P, Huerta A. Error estimation and adaptivity in strain localization problems with softening materials. Monograph CIMNE no. 40, Barcelona, 1997.

28. Strouboulis T, Haque KA. Recent experiences with error estimation and adaptivity, Part I: Review of error estimators for scalar elliptic problems. Computer Methods in Applied Mechanics and Engineering 1992; 97:399-436.

29. Strouboulis T, Haque KA. Recent experiences with error estimation and adaptivity, Part II: Error estimation for $h$-adaptive approximations on grids of triangles and quadrilaterals. Computer Methods in Applied Mechanics and Engineering 1992; 100:359-430.

30. Cirak F, Ramm E. A-posteriori error estimation and adaptivity for linear elasticity using the reciprocal theorem. Computer Methods in Applied Mechanics and Engineering 1998; 156:351-362.

31. Díez P, Egozcue JJ, Huerta A. A posteriori error estimation for standard finite element analysis. Computer Methods in Applied Mechanics and Engineering 1998; 163:141-157.

32. Huerta A, Díez P. Error estimation including pollution assessment for nonlinear finite element analysis. Computer Methods in Applied Mechanics and Engineering 2000; 181:21-41.

33. Díez P, Egozcue JJ, Huerta A. Analysis of the average efficiential of an error estimator. In Finite Element Methods: Superconvergence, Post-processing and a Posteriori Error Estimates, Kř́žzek M. et al. (eds). Marcel Dekker: New York, 1997; 113-126.

34. Bank RE, Weiser A. Some a posteriori error estimators for elliptic partial differential equations. Mathematics of Computation 1985; 44:283-301.

35. Ainsworth M, Oden JT. A unified approach to a posteriori error estimation using element residual methods. Numerische Mathematik 1993; 65:23-50.

36. Ladevèze P, Pelle JP, Rougeot Ph. Error estimation and mesh optimization for classical finite elements. Engineering Computations 1991; 8:69-80.

37. Hughes TJR. The Finite Element Method. Prentice-Hall International: Stanford, 1987.

38. Wahlbin LB. Local behavior in finite element methods. In Handbook of Numerical Analysis, vol. II, Ciarlet PG, Lions JL (eds). North-Holland: Amsterdam, 1991; 357-522.

39. Zienkiewicz OC, Zhu JZ. A simple error estimator and adaptive procedure for practical engineering analysis. International Journal for Numerical Methods in Engineering 1987; 24:337-357.

40. Oñate E, Bugeda G. A study of mesh optimality criteria in adaptive finite element analysis. Engineering Computations 1993; 10:307-321.

41. Li LY, Bettess P. Notes on mesh optimal criteria in adaptive finite element computations. Communications in Numerical Methods in Engineering 1995; 11:911-915.

42. Díez P, Huerta A. A unified approach to remeshing strategies for finite element h-adaptivity. Computer Methods in Applied Mechanics and Engineering 1999; 176:215-229.

43. Sarrate J. Modelización numérica de la interacción fluido-sólido rígido: desarrollo de algoritmos, generación de mallas y adaptividad. Doctoral Thesis, Universitat Politècnica de Catalunya, Barcelona, 1996. 